\title{
The Euler characteristic correction to the Kähler potential - revisited
}

\author{
Federico Bonetti ${ }^{a}$ and Matthias Weissenbacher ${ }^{b}$ \\ ${ }^{a}$ C.N. Yang Institute for Theoretical Physics, SUNY Stony Brook, \\ Stony Brook, New York 11794, U.S.A. \\ ${ }^{b}$ Kavli Institute for the Physics and Mathematics of the Universe, University of Tokyo, \\ Kashiwa-no-ha 5-1-5, 277-8583, Japan \\ E-mail: bonetti@insti.physics.sunysb.edu, \\ matthias.weissenbacher@ipmu.jp
}

Abstract: We confirm the leading $\alpha^{\prime 3}$ correction to the $4 \mathrm{~d}, \mathcal{N}=1$ Kähler potential of type IIB orientifold compactifications, proportional to the Euler characteristic of the Calabi-Yau threefold (BBHL correction). We present the explicit solution for the $\alpha^{\prime 3}$ modified internal background metric in terms of the non-harmonic part of the third Chern form of the leading order Calabi-Yau manifold. The corrected internal manifold is almost Calabi-Yau and admits an SU(3) structure with non-vanishing torsion. We also find that the full ten-dimensional Einstein frame background metric is multiplied by a non-trivial Weyl factor. Performing a Kaluza-Klein reduction on the modified background we derive the $\alpha^{\prime 3}$-corrected kinetic terms for the dilaton and the Kähler deformations of the internal Calabi-Yau threefold for arbitrary $h^{1,1}$. We analyze these kinetic terms in the $4 \mathrm{~d}, \mathcal{N}=2$ un-orientifolded theory, confirming the expected correction to the Kähler moduli space prepotential, as well as in the $4 \mathrm{~d}, \mathcal{N}=1$ orientifolded theory, thus determining the corrections to the Kähler potential and Kähler coordinates.

KEYWords: Superstring Vacua, Superstrings and Heterotic Strings

ARXIV EPRINT: 1608.01300 


\section{Contents}

1 Introduction 1

2 Four-dimensional $\alpha^{\prime 3}$ Lagrangian $\quad 3$

2.1 Type IIB higher-derivative action 3

2.2 Supersymmetric background 5

2.2.1 Corrections to the background 5

2.2.2 $\mathrm{SU}(3)$ structure on the internal manifold $\quad 8$

2.2.3 Fluctuations associated to Kähler moduli 9

$\begin{array}{lll}2.3 & \text { Reduction results } & 10\end{array}$

$\begin{array}{lll}2.3 .1 & \text { Results and uplift } & 11\end{array}$

$\begin{array}{lll}2.3 .2 & \text { Weyl rescaling } & 14\end{array}$

3 Correction to the $\mathcal{N}=2$ prepotential $\quad 14$

3.1 Translation into $\mathcal{N}=2$ field variables $\quad 14$

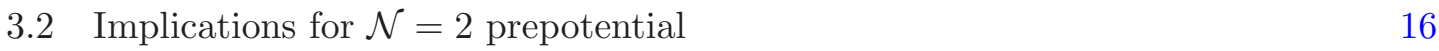

$4 \mathcal{N}=1$ Kähler coordinates and Kähler potential $\quad 17$

$\begin{array}{lll}4.1 & \text { Correction to the Kähler potential and coordinates } & 17\end{array}$

4.2 Some comments 20
2.17

5 Conclusions $\quad 21$

$\begin{array}{ll}\text { A Conventions, definitions, and identities } & 22\end{array}$

B Higher-derivative dilaton terms $\quad 24$

\section{Introduction}

Supersymmetric flux compactifications of type IIB superstring theory constitute a vast and rich arena for the study of the implications of string dynamics on $4 \mathrm{~d}$ physics. Comprehensive reviews on the subject include [1-5]. A common feature of several flux compactification scenarios is the crucial role played by $\alpha^{\prime}$ and/or $g_{s}$ corrections to the leading-order effective action. One of the most prominent perturbative corrections to the $4 \mathrm{~d}$ low-energy effective action is the $\alpha^{\prime 3}$ correction to the Kähler potential proportional to the Euler characteristic of the Calabi-Yau threefold used in the compactification. This correction was first computed in a paper by Becker, Becker, Haack, and Louis (BBHL) [6]. It plays in essential role in the Large Volume Scenario for type IIB compactifications [7-9]. 
The 10d origin of the Euler characteristic correction to the $4 \mathrm{~d}$ Kähler potential resides in the leading $\alpha^{\prime}$ corrections to the bulk type IIB supergravity action. This is an eightderivative coupling built with four Riemann tensors, accompanied by several additional couplings involving other type IIB fields as a consequence of $\mathrm{SL}(2, \mathbb{Z})$ symmetry. For the derivation and discussion of these couplings see e.g. [10-19]. The authors of [6] infer the corresponding correction to the 4d Kähler potential in a somewhat indirect way. Exploiting mirror symmetry, the starting point of their analysis are the results of [20, 21] about the corrected metric of the hypermultiplet moduli space of a Calabi-Yau type II compactification to four dimensions with $\mathcal{N}=2$ supersymmetry. Making use of the results of [22] these corrections are then reformulated in terms of $4 \mathrm{~d}$ field variables whose $10 \mathrm{~d}$ origin is manifest, in such a way that the orientifold projection to $\mathcal{N}=1$ supersymmetry can be performed.

The aim of this paper is to provide a direct derivation of the Euler characteristic correction to the 4d Kähler potential by means of a completely explicit Kaluza-Klein reduction of the relevant $\alpha^{\prime 3}$ couplings in the 10d bulk action. Not only is this approach more transparent, but it also presents other advantages. Firstly, we do not have to make any assumption about the superpotential or the scalar potential of the full $4 \mathrm{~d}, \mathcal{N}=1$ low-energy effective action, since our conclusions are entirely drawn from the examination of the kinetic terms for the dilaton and the Kähler moduli of the Calabi-Yau threefold for arbitrary $h^{1,1}$. Secondly, our approach allows us to derive both the correction to the $4 \mathrm{~d}$ Kähler potential and the correction to the Kähler coordinates as a function of the Kähler moduli. Finally, in the process of the derivation one necessarily has to analyze the background solution and show how it gets modified by the $\alpha^{\prime}$ corrections under examination. In particular, we are able to provide an explicit solution for the $\alpha^{\prime}$-corrected internal metric in terms of the non-harmonic part of the third Chern form $c_{3}$ of the leading order Calabi-Yau threefold background. The corrected metric is Kähler but not Ricci-flat. As a result it no longer has $\mathrm{SU}(3)$ holonomy, but rather an $\mathrm{SU}(3)$ structure with non-vanishing torsion. The corrected geometry fits in the framework of almost Calabi-Yau manifolds [23]. Similar results were derived in [24] in the case of M-theory compactifications on a Calabi-Yau fourfold. Additionally, we find that the 10d background metric is corrected by an overall Weyl factor at order $\alpha^{\prime 3}$, in analogy with the findings of the three-dimensional M-theory analysis of [24-28]. Throughout this paper, we let higher derivatives act in the internal space, working at two-derivative level in the external directions. Recently, $\alpha^{\prime 3}$ external four-derivative couplings between Kähler moduli and gravity were derived in [29] for the same class of type IIB orientifold setups considered in this work, with potential applications to Kähler moduli inflation studies.

The main results of our paper are summarized in equations (4.18) to (4.21). We reproduce the Euler characteristic correction to the Kähler potential originally found by BBHL [6]. We also compute the leading $\alpha^{\prime 3}$ corrections to the Kähler coordinates and the $4 \mathrm{~d}$ axio-dilaton in terms of the Kähler moduli, and find that they are vanishing. Finally, we exclude the possibility that the effect of the correction to the Kähler potential can be undone by a Kähler coordinate redefinition, and we reformulate our findings in the formalism of linear multiplets [30-32] in order to elucidate the physical relevance of the correction. Let us remark that our analysis does not take into account explicitly localized sources of the $\mathcal{N}=1$ setup, such as seven-branes and orientifold planes. 
This paper is organized as follows. Section 2 is devoted to the computation of the relevant $\alpha^{\prime}$ corrections to the $4 \mathrm{~d}$ kinetic terms of the dilaton and Kähler moduli. In particular, in section 2.1 we present the relevant higher-derivative corrections to the $10 \mathrm{~d}$ action of type IIB supergravity, in section 2.2 we discuss the background solutions of interest, while section 2.3 is devoted to the dimensional reduction to four dimensions. The results of the computation are then analyzed in section 3 in the context of the $4 \mathrm{~d}, \mathcal{N}=2$ effective theory obtained in the absence of orientifold planes and D-branes. We show that our findings are compatible with the expected perturbative correction to the prepotential for the Kähler moduli space metric. We then proceed in section 4 to analyze the $4 \mathrm{~d}, \mathcal{N}=1$ setup obtained after implementation of an orientifold projection. We verify that the $\alpha^{\prime}$-corrected kinetic terms of the dilaton and Kähler moduli can be written in terms of a corrected Kähler potential. We determine the latter, reproducing the correction of BBHL [6], and we identify the $\alpha^{\prime}$-corrected form of the Kähler coordinates. Section 5 summarizes our conclusions. Our conventions, together with useful identities and some technical material, can be found in the appendices.

\section{Four-dimensional $\alpha^{3}$ Lagrangian}

This section discusses the dimensional reduction of IIB supergravity action including a suitable class of eight-derivative corrections, on a Calabi-Yau threefold to four dimensions. In particular, we restrict our analysis to purely gravitational terms and dilaton terms. We fluctuate the background metric by Kähler deformations and focus on couplings that carry two external spacetime derivatives and are at most quadratic in the infinitesimal Kähler deformations. We first review the relevant eight-derivative $\alpha^{\prime 3}$ corrections to $10 \mathrm{~d}$ type IIB supergravity and the supersymmetric background.

\subsection{Type IIB higher-derivative action}

The starting point of our analysis consists of the type IIB supergravity action including the leading order $\alpha^{\prime 3}$ eight-derivative correction built with four Riemann tensors [10-19]. For our purposes this gravitation coupling has to be supplemented with suitable dilaton couplings discussed below.

In order to set up our notation, let us first record the gravitational and axio-dilaton terms in the two-derivative type IIB supergravity in the Einstein frame,

$$
S_{R, \tau}^{(2)}=\frac{1}{2 \kappa_{10}^{2}} \int\left(R-\frac{1}{2 \tau_{2}^{2}} \nabla_{M} \tau \nabla^{M} \bar{\tau}\right) *_{10} 1,
$$

where $2 \kappa_{10}^{2}=(2 \pi)^{7} \alpha^{\prime 4}, M=0, \ldots, 9$ is a $10 \mathrm{~d}$ world index, and the axio-dilaton is defined as

$$
\tau=\tau_{1}+i \tau_{2}=C_{0}+i e^{-\hat{\phi}} .
$$

The action $(2.1)$ is invariant under the $\mathrm{SL}(2, \mathbb{Z})$ symmetry of type IIB superstring theory, under which the Einstein frame metric is invariant and the axio-dilaton transforms as

$$
\tau^{\prime}=\frac{a \tau+b}{c \tau+d}, \quad\left(\begin{array}{ll}
a & b \\
c & d
\end{array}\right) \in \mathrm{SL}(2, \mathbb{Z}) .
$$


Next, let us consider the $\alpha^{\prime 3}$ correction constructed with four Riemann tensors. It takes the form

$$
S_{R^{4}}^{(8)}=\frac{1}{2 \kappa_{10}^{2}} \cdot \frac{\alpha^{\prime 3}}{3 \cdot 2^{12}} \int f_{0}(\tau, \bar{\tau})\left(t_{8} t_{8}+\frac{1}{8} \epsilon_{10} \epsilon_{10}\right) R^{4} *_{10} 1
$$

where the explicit tensor contractions are given by

$$
\begin{aligned}
\epsilon_{10} \epsilon_{10} R^{4} & =\epsilon^{R_{1} R_{2} M_{1} \ldots M_{8}} \epsilon_{R_{1} R_{2} N_{1} \ldots N_{8}} R^{N_{1} N_{2}} M_{1} M_{2} R^{N_{3} N_{4}}{ }_{M_{3} M_{4}} R^{N_{5} N_{6}}{ }_{M_{5} M_{6}} R^{N_{7} N_{8}} M_{7} M_{8}, \\
t_{8} t_{8} R^{4} & =t_{8}^{M_{1} \ldots M_{8}} t_{8} N_{1} \ldots N_{8} R^{N_{1} N_{2}}{ }_{M_{1} M_{2}} R^{N_{3} N_{4}}{ }_{M_{3} M_{4}} R^{N_{5} N_{6}}{ }_{M_{5} M_{6}} R^{N_{7} N_{8}}{ }_{M_{7} M_{8}},
\end{aligned}
$$

and the tensor $t_{8}$ is defined in terms of the metric in the standard way [12]. We have also introduced the function $f_{0}(\tau, \bar{\tau})$, which is the non-holomorphic, $\operatorname{SL}(2, \mathbb{Z})$-invariant function defined as

$$
f_{0}(\tau, \bar{\tau})=\sum_{(m, n) \neq(0,0)} \frac{\tau_{2}^{3 / 2}}{|m+n \tau|^{3}} .
$$

It is useful to note that in the large $\tau_{2}$ limit, which corresponds to the small string coupling limit, we have

$$
f_{0}(\tau, \bar{\tau})=2 \zeta(3) \tau_{2}^{3 / 2}+\frac{2 \pi^{2}}{3} \tau_{2}^{-1 / 2}+\mathcal{O}\left(e^{-2 \pi \tau_{2}}\right) .
$$

Our analysis requires the consideration of additional higher-derivative terms involving gradients of the dilaton. Such terms can be obtained following the approach of $[14,17,18]$, by replacing each occurrence of the Riemann tensor in (2.5) according to

$$
R_{M N}^{P Q} \rightarrow R_{M N}^{P Q}+\tilde{c}_{1} g_{[M}^{[P} \nabla_{N]} \nabla^{Q]} \hat{\phi}+\tilde{c}_{2} g_{[M}^{[P} \nabla_{N]} \hat{\phi} \nabla^{Q]} \hat{\phi}+\tilde{c}_{3} g_{[M}^{[P} g_{N]}{ }^{Q]} \nabla_{K} \phi \nabla^{K} \hat{\phi}
$$

where antisymmetrizations are performed with weight one, and $\tilde{c}_{1}, \tilde{c}_{2}, \tilde{c}_{3}$ are real numerical coefficients that we leave unfixed for now. ${ }^{1}$ In order to achieve $\operatorname{SL}(2, \mathbb{Z})$-invariance several other terms have to be added to the action [33, 34], but they are not relevant for our discussion.

In summary, the total action utilized in the analysis of the following sections is obtained by summing the two-derivative terms in (2.1) with the terms generated by the replacement rule (2.8) in the terms given in (2.5). We only keep terms which can correct the kinetic terms of the resulting $4 \mathrm{~d}$ dilaton and discarding higher derivative terms thereof, as well as terms for the axion $C_{0}$. We also retain only the leading term in the large $\tau_{2}$ expansion of $f_{0}(\tau, \bar{\tau})$, see $(2.7)$. We thus obtain

$$
\begin{aligned}
S= & \frac{1}{2 \kappa_{10}^{2}} \int\left[R-\frac{1}{2} \nabla_{M} \phi \nabla^{M} \phi+\frac{\zeta(3) \alpha^{\prime 3}}{3 \cdot 2^{11}} e^{-\frac{3}{2} \phi}\left(t_{8} t_{8}+\frac{1}{8} \epsilon_{10} \epsilon_{10}\right) R^{4}\right. \\
& \left.+\frac{\zeta(3) \alpha^{\prime 3}}{3 \cdot 2^{11}} e^{-\frac{3}{2} \phi}\left(\tilde{c}_{1} \nabla^{M} \nabla_{M} \hat{\phi} f_{1}+\tilde{c}_{2} \nabla_{M} \hat{\phi} \nabla_{N} \hat{\phi} f_{2}^{M N}+\tilde{c}_{3} \nabla^{M} \hat{\phi} \nabla_{M} \hat{\phi} f_{3}\right)\right] *_{10} 1,
\end{aligned}
$$

${ }^{1}$ The analysis of the 4-point tree-level dilaton scattering amplitude gives the value $\tilde{c}_{1}=-1$ (see $[14,17,18]$ and also $[33,34]$ ), while $\tilde{c}_{2}, \tilde{c}_{3}$ cannot be fixed in this way. In what follows, however, we proceed treating all $\tilde{c}$ coefficients on the same footing. 
where the quantities $f_{1}, f_{2}^{M N}, f_{3}$ are homogeneous polynomials of degree three in the components of the Riemann tensor and are given explicitly in appendix B. For notational convenience let us introduce the dimensionful constant

$$
\alpha=\frac{\zeta(3) \alpha^{\prime 3}}{3 \cdot 2^{11}}
$$

which plays the role of the small expansion parameter relevant for our problem. Note that we adopt conventions in which the $10 \mathrm{~d}$ metric components and the dilaton are dimensionless, while coordinates $x^{M}$ have dimension of length.

\subsection{Supersymmetric background}

In this section we study how the supersymmetric Calabi-Yau background solutions of type IIB supergravity are modified in the presence of the higher-derivative terms in (2.9) and we present the relevant dilaton and Kähler fluctuations around the corrected background. Let us note that we cannot analyze directly the supersymmetry properties of the background, since the type IIB supersymmetry variations are not completely known at the required order in $\alpha^{\prime}$, but we can give necessary conditions for the $\alpha^{\prime}$ modification of the background by solving the equations of motion.

\subsubsection{Corrections to the background}

Our problem fits into the framework of supersymmetric flux compactifications of type IIB superstring on a Calabi-Yau threefold $Y_{3}$. Let us first recall some facts about these setups neglecting higher-derivative corrections to the 10d supergravity action [35]. For compactifications to flat four-dimensional spacetime the background metric has the form

$$
d s_{10}^{2}=e^{2 A} \eta_{\mu \nu} d x^{\mu} d x^{\nu}+e^{-2 A} g_{a b}^{(0)} d y^{a} d y^{b}
$$

where $\mu, \nu=0,1,2,3$ are $4 \mathrm{~d}$ external spacetime world indices, $x^{\mu}$ are Cartesian coordinates, $\eta_{\mu \nu}$ is the Minkowski metric, $a, b=1, \ldots 6$ are real internal world indices associated to the coordinates $y^{a}$, and $g_{a b}^{(0)}$ denotes the Ricci-flat metric on $Y_{3}$. The warp factor $A$ only depends on the internal coordinates and also determines the background $F_{5}$ flux via

$$
F_{5}=\left(1+*_{10}\right) d e^{4 A} \wedge d x^{0} \wedge d x^{1} \wedge d x^{3} \wedge d x^{4}
$$

The $F_{5}$ flux has to obey the Bianchi identity

$$
d F_{5}=H_{3} \wedge F_{3}+\rho_{6}
$$

where $H_{3}=d B_{2}$ and $F_{3}=d C_{2}-C_{0} d B_{2}$ are the usual NSNS and RR three-form field strengths and the six-form $\rho_{6}$ encodes the D3-brane charge density associated with possible localized sources. Integrating (2.13) on the internal manifold yields the D3-brane tadpole cancellation condition

$$
\frac{1}{2 \kappa_{10}^{2} T_{3}} \int_{Y_{3}} H_{3} \wedge F_{3}+Q_{\mathrm{D} 3}=0
$$


where $T_{3}=(2 \pi)^{-3}\left(\alpha^{\prime}\right)^{-2}$ is the D3-brane tension and $Q_{\mathrm{D} 3}$ is the total D3-brane charge, proportional to the integral of $\rho_{6} .{ }^{2}$ If all local sources are removed, all fluxes have to vanish and the warp factor is necessarily trivial.

The inclusion of higher-derivative corrections to the type IIB bulk action induces modifications in the previous picture. In what follows we analyze the $10 \mathrm{~d}$ equations of motion in presence of higher-derivative corrections of order $\alpha \propto \alpha^{\prime 3}$ but without introducing any local source in the problem. Even though this setup yields an effective $4 \mathrm{~d}, \mathcal{N}=2$ lowenergy effective action, we argue that the following analysis is sufficient for the purpose of studying the Euler characteristic correction to the Kähler potential in the $4 \mathrm{~d}, \mathcal{N}=1$ theory after performing the orientifold truncation in section 4.

We adopt the following $\alpha^{\prime}$-corrected Ansatz for the 10d background metric,

$$
d s_{10}^{2}=e^{\Phi}\left[e^{2 A} \eta_{\mu \nu} d x^{\mu} d x^{\nu}+e^{-2 A} g_{a b} d y^{a} d y^{b}\right]
$$

where

$$
\begin{aligned}
\Phi & =\alpha \Phi^{(1)}+\mathcal{O}\left(\alpha^{2}\right), \\
A & =\alpha A^{(1)}+\mathcal{O}\left(\alpha^{2}\right) \\
g_{a b} & =g_{a b}^{(0)}+\alpha g_{a b}^{(1)}+\mathcal{O}\left(\alpha^{2}\right) .
\end{aligned}
$$

The quantity $\Phi$ is an overall 10d Weyl rescaling of the metric, and it has been introduced in analogy with the analysis of [24]. This parametrization of the prefactors multiplying the internal and external metric is general and is useful for the following discussion. The zeroth-order metric is the Calabi-Yau Ricci-flat metric. Accordingly, $\Phi$ and $A$ have no $\mathcal{O}\left(\alpha^{0}\right)$ term. All quantities depend exclusively on the internal coordinates in order not to break Poincaré invariance in the external directions. The corrections to the dilaton profile are parametrized as

$$
\hat{\phi}=\phi_{0}+\alpha \phi^{(1)}+\mathcal{O}\left(\alpha^{2}\right),
$$

where $\phi_{0}$ is the constant uncorrected dilaton VEV.

The 10d Einstein equation at order $\alpha$ can be written in the form

$$
0=R_{M N}^{(1)}-\frac{1}{2}\left(R_{P Q}^{(1)} g^{(0) P Q}\right) g_{M N}^{(0)}+\mathcal{T}_{M N}
$$

The first two terms capture the contribution coming from the two-derivative part of the Einstein equation evaluated on the $\alpha$-corrected Ansatz (2.15). The symbol $R_{M N}^{(1)}$ is used to denote the $\mathcal{O}(\alpha)$ part of the 10d Ricci tensor computed using the metric (2.15), while $g_{M N}^{(0)}$ is used for the $\mathcal{O}\left(\alpha^{0}\right)$ part of $(2.15)$. The quantity $\mathcal{T}_{M N}$ encodes all the contributions coming from the higher-derivative part of the Einstein equation, derived from (2.9), upon evaluation on the $\mathcal{O}\left(\alpha^{0}\right)$ part of the metric Ansatz (2.15). We find

$$
\mathcal{T}_{\mu \nu}=0, \quad \mathcal{T}_{\mu a}=0, \quad \mathcal{T}_{a b}=768 \alpha e^{-\frac{3}{2} \phi_{0}} J^{(0)}{ }_{a}^{c} J^{(0)}{ }_{b}^{d} \nabla_{c}^{(0)} \nabla_{d}^{(0)} Q
$$

\footnotetext{
${ }^{2}$ Recall that $Q_{\mathrm{D} 3}$ generically receives contributions not only from $D 3$-branes and $O 3$-planes, but also from higher-dimensional defects with flux- and/or geometry-induced $D 3$-brane charge, such as $D 7$-branes and O7-planes [35].
} 
where $\nabla^{(0)}, J^{(0)}$ denote the Levi-Civita connection and complex structure associated to the zeroth-order metric, respectively, and the quantity $Q$ is the six-dimensional Euler density,

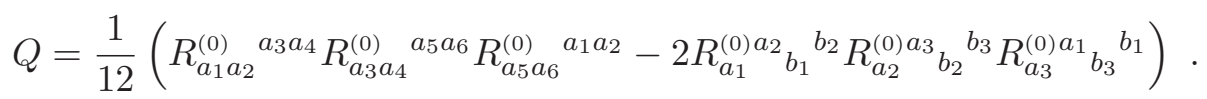

This object satisfies

$$
Q=(2 \pi)^{3} *_{6}^{(0)} c_{3}^{(0)}, \quad \int Q *_{6}^{(0)} 1=(2 \pi)^{3} \chi,
$$

where $\chi$ is the Euler characteristic of the internal space, $*_{6}^{(0)}$ is the Hodge star operator with respect to the zeroth-order metric, and $c_{3}$ is the third Chern form built from $g_{a b}^{(0)}$, defined in appendix A.

It is convenient to use holomorphic and antiholomorphic indices $m=1,2,3, \bar{m}=\overline{1}, \overline{2}, \overline{3}$ associated to the zeroth-order complex structure, in such a way that $J^{(0)}{ }_{m}^{n}=+i \delta_{m}{ }^{n}$. One may then check that all components of the order- $\alpha$ Einstein's equation are solved by imposing

$$
\Phi^{(1)}=-192 e^{-\frac{3}{2} \phi_{0}} Q, \quad R_{m \bar{n}}^{(1)}=-1536 e^{-\frac{3}{2} \phi_{0}} \nabla_{m}^{(0)} \nabla_{\bar{n}}^{(0)} Q, \quad R_{m n}^{(1)}=0, \quad R_{\bar{m} \bar{n}}^{(1)}=0, \quad A^{(1)}=0,
$$

where $R^{(1)}$ denotes the linearized Ricci tensor of the metric correction $g^{(1)}$. Let us also point out that we can exhibit an explicit expression for $g^{(1)}$. To this end we start recalling that, as a consequence of the $\partial \bar{\partial}$-lemma, the $(3,3)$-form $c_{3}$ can be decomposed as

$$
c_{3}=H c_{3}+i \partial \bar{\partial} F
$$

where $H c_{3}$ denotes the harmonic part of $c_{3}$ with respect to the zeroth-order metric and $F$ is a suitable co-closed $(2,2)$-form. ${ }^{3}$ We can express the Euler density $Q$ in terms of $F$ as

$$
(2 \pi)^{-3} Q=*_{6}^{(0)} H c_{3}+\frac{1}{2} \Delta^{(0)} *_{6}^{(0)}\left(J^{(0)} \wedge F\right),
$$

where $\Delta^{(0)}=2 g^{(0) m \bar{n}} \nabla_{m}^{(0)} \nabla_{\bar{n}}^{(0)}$ denotes the scalar Laplacian of the zeroth-order metric, and we exploited the co-closure of $F$. Let us also stress that the first term in (2.24) is constant on the threefold as a result of the harmonic projection. Utilizing (2.24) one can observe that the equations for $R^{(1)}$ in $(2.22)$ can be solved by setting

$$
g_{m n}^{(1)}=0, \quad g_{\bar{m} \bar{n}}^{(1)}=0, \quad g_{m \bar{n}}^{(1)}=-1536(2 \pi)^{3} e^{-\frac{3}{2} \phi_{0}} \nabla_{m}^{(0)} \nabla_{\bar{n}}^{(0)} *_{6}^{(0)}\left(J^{(0)} \wedge F\right) .
$$

\footnotetext{
${ }^{3}$ The existence and co-closure of $F$ can be seen by the following argument, similar to an argument in [36]. Let us first apply the Hodge decomposition theorem to the 6 -form $c_{3}$ and write $c_{3}=H c_{3}+d b_{5}$ for a suitable globally defined 5 -form $b_{5}$. Since the form $d b_{5}$ is $d$-exact, $\partial$-closed, and $\bar{\partial}$-closed, the $\partial \bar{\partial}$-lemma ensures that it is $\partial \bar{\partial}$-exact, so that $d \beta_{5}=\partial \bar{\partial} f_{4}$ for a globally defined 4-form $f_{4}$. We can now apply the Hodge decomposition theorem to $f_{4}$ and write $f_{4}=H f_{4}+d g_{3}+\delta g_{5}$, where $\delta$ denotes the codifferential and $g_{3}, g_{5}$ are a globally defined 3 - and 5 -form, respectively. We now note that $H f_{4}$ is harmonic on a Kähler manifold and hence $\bar{\partial}$-closed, and that $\partial \bar{\partial} d=0$. It follows that we can write $c_{3}=H c_{3}+\partial \bar{\partial} \delta g_{5}$. We can thus set $F=-i \delta g_{5}$, which is co-closed because it is co-exact.
} 
As we can see, the corrected metric $g_{m \bar{n}}^{(0)}+\alpha g_{m \bar{n}}^{(1)}$ is still Kähler and belongs to the same Kähler class as $g_{m \bar{n}}^{(0)}$.

The dilaton also receives a correction sourced by the higher-derivative terms in (2.9). The relevant terms in the dilaton equation of motion yield the relation

$$
0=g^{(0) \bar{n} m} \nabla_{m}^{(0)} \nabla_{\bar{n}}^{(0)} \phi^{(1)}-1152 e^{-\frac{3}{2} \phi_{0}} \tilde{c}_{1} g^{(0) \bar{n} m} \nabla_{m}^{(0)} \nabla_{\bar{n}}^{(0)} Q
$$

and is solved by

$$
\phi^{(1)}=1152 \tilde{c}_{1} e^{-\frac{3}{2} \phi_{0}} Q
$$

An integration constant has been set to zero because, up to $\mathcal{O}\left(\alpha^{2}\right)$ terms, it can always be reabsorbed in $\phi_{0}$. Our findings about the modification of the background dilaton profile and internal metric are in line with previous work [13, 37-40].

In closing this section let us remark that a full determination of $F_{1}, G_{3}, F_{5}$ at order $\alpha$ would require a detailed knowledge of order- $\alpha$ corrections to their equations of motion, which are related to the $\mathrm{SL}(2, \mathbb{Z})$ completion of the higher-derivative terms recorded in (2.9). This investigation is beyond the scope of the present work. Since we focus on couplings involving the metric and the dilaton, however, we only need the expressions (2.22) and (2.27) for the order- $\alpha$ Weyl rescaling factor and dilaton correction.

\subsubsection{SU(3) structure on the internal manifold}

In this section we show how the SU(3) holonomy of the zeroth-order Calabi-Yau internal metric is deformed to a specific $\mathrm{SU}(3)$ structure of the $\alpha^{\prime 3}$-modified background. Our discussion applies to $g_{a b}^{(0)}+\alpha g_{a b}^{(1)}$ and does not involve the overall Weyl rescaling factor of the $10 \mathrm{~d}$ metric.

The Kähler form and the (3,0)-form of the uncorrected Calabi-Yau metric are subject to $\alpha^{\prime}$ corrections but remain nonetheless globally defined on the internal space. As a result, the structure group of the internal manifold is reduced from $\mathrm{SO}(6)$ to $\mathrm{SU}(3)$ even after taking corrections into account. This guarantees the existence of local $\mathrm{SU}(3)$ coframes, consisting of triplets $e^{I}, I=1,2,3$, of complex one-forms together with their complex conjugates $\bar{e}^{\bar{I}}$, with the property that $\left\{e^{I}, \bar{e}^{\bar{I}}\right\}$ is a local basis of $T^{*} Y_{3} \otimes \mathbb{C}$ and on overlapping patches the transition functions for $e^{I}$ take values in $\mathrm{SU}(3)$. We may express locally $J$ and $\Omega$, as well as the metric, in terms of the $\mathrm{SU}(3)$ coframe as

$$
J=i \delta_{I \bar{J}} e^{I} \wedge \bar{e}^{\bar{J}}, \quad \Omega=\frac{1}{3 !} \epsilon_{I J K} e^{I} \wedge e^{J} \wedge e^{K}, \quad d s^{2}=2 \delta_{I \bar{J}} e^{I} \bar{e}^{\bar{J}},
$$

where $\epsilon_{123}=1$. The coframe can be written as the sum of an uncorrected contribution and a correction,

$$
e^{I}=e^{(0) I}+\alpha e^{(1) I} .
$$

Since the uncorrected geometry is Kähler, we can adopt complex coordinates and choose the coframe $e^{(0) I}$ in such a way that its only non-zero components are $e^{(0) I}{ }_{m}, \bar{e}^{(0) \bar{I}} \bar{n}$.

The relevant correction to the $\mathrm{SU}(3)$ coframe takes the form

$$
e^{(1) I}=-768(2 \pi)^{3} e^{-\frac{3}{2} \phi_{0}} e^{(0) I}{ }_{n} \nabla_{m}^{(0)} \nabla^{(0) n} *_{6}^{(0)}\left(J^{(0)} \wedge F\right) d z^{m} .
$$


It is indeed straightforward to check that the associated correction to the metric reproduces $g^{(1)}$ in (2.25). We also have

$$
J=J^{(0)}+\alpha J^{(1)}, \quad J^{(1)}=-1536 i(2 \pi)^{3} e^{-\frac{3}{2} \phi_{0}} \partial^{(0)} \bar{\partial}^{(0)} *_{6}^{(0)}\left(J^{(0)} \wedge F\right),
$$

and therefore

$$
d J^{(1)}=0 .
$$

The correction to the $(3,0)$ form reads

$$
\Omega=\Omega^{(0)}+\alpha \Omega^{(1)}, \quad \Omega^{(1)}=\left[-384(2 \pi)^{3} e^{-\frac{3}{2} \phi_{0}} \Delta^{(0)} *_{6}^{(0)}\left(J^{(0)} \wedge F\right)\right] \Omega^{(0)},
$$

which implies

$$
d \Omega^{(1)}=-768(2 \pi)^{3} e^{-\frac{3}{2} \phi_{0}} d Q \wedge \Omega^{(0)} .
$$

We can summarize our conclusions in the language of SU(3) torsion classes, reviewed for instance in [41]. The most general SU(3) structure can be described by

$$
\begin{aligned}
& d J=-\frac{3}{2} \operatorname{Im}\left(\mathcal{W}_{1} \bar{\Omega}\right)+\mathcal{W}_{4} \wedge J+\mathcal{W}_{3}, \\
& d \Omega=\mathcal{W}_{1} J \wedge J+\mathcal{W}_{2} \wedge J+\overline{\mathcal{W}}_{5} \wedge \Omega,
\end{aligned}
$$

with suitable torsion classes $\mathcal{W}_{1}, \mathcal{W}_{2}, \mathcal{W}_{3}, \mathcal{W}_{4}, \overline{\mathcal{W}}_{5}$. We can then see that in our case all torsion classes vanish, with the exception of

$$
\overline{\mathcal{W}}_{5}=0+\alpha \overline{\mathcal{W}}_{5}^{(1)}, \quad \overline{\mathcal{W}}_{5}^{(1)}=-768(2 \pi)^{3} e^{-\frac{3}{2} \phi_{0}} \bar{\partial}^{(0)} Q .
$$

The corrected geometry is still Kähler and the manifold is an almost Calabi-Yau threefold [23]. The situation at hand can be compared to considering, for instance, a quintic in $\mathbb{P}^{4}$ endowed with the metric induced by the ambient space Fubini-Study metric. It is interesting to point out, however, that if the requirement of simple connectedness is relaxed, it is possible to have Ricci-flat Kähler manifolds that are almost Calabi-Yau, but not Calabi-Yau. An example is furnished by the Enriques surfaces; recently, novel examples have been constructed in [42] in the context of solvmanifolds.

\subsubsection{Fluctuations associated to Kähler moduli}

In order to derive the relevant couplings in the four-dimensional effective action of type IIB compactified on $Y_{3}$ we need to activate Kähler structure deformations of the internal metric entering the 10d background solution. We thus imagine to pick a fixed, reference point in the complex structure and Kähler structure moduli space of $Y_{3}$ and to switch on small deformations in the Kähler structure moduli space directions.

It is well-known that, at zeroth-order in $\alpha$, the Kähler structure deformations of the internal Ricci-flat metric take the form

$$
\delta g_{m \bar{n}}^{(0)}=-i \delta v^{i} \omega_{i m \bar{n}}
$$

where $i=1, \ldots, h^{1,1}\left(Y_{3}\right), \delta v^{i}$ are real deformation parameters, and $\omega_{i m \bar{n}}$ denote a basis of harmonic $(1,1)$-forms whose cohomology classes are Poincaré dual to an integral basis of the homology $H_{4}\left(Y_{3}, \mathbb{Z}\right)$. 
At first order in $\alpha$ the structure of the internal metric deformations can be written as

$$
\delta\left(g_{m \bar{n}}^{(0)}+\alpha g_{m \bar{n}}^{(1)}\right)=-i \delta v^{i}\left(\omega_{i m \bar{m}}+\alpha \nabla_{m}^{(0)} \nabla_{\bar{n}}^{(0)} \rho_{i}\right)+\alpha \nabla_{m}^{(0)} \nabla_{\bar{n}}^{(0)} \delta \tilde{F}+\mathcal{O}\left(\alpha^{2}\right)
$$

The $\rho_{i}$ functions parametrize deviations from the harmonic representative within each cohomology class in $H^{1,1}\left(Y_{3}\right)$, while $\delta \tilde{F}$ denotes the variation of the function $\tilde{F}=*_{6}^{(0)}\left(J^{(0)} \wedge\right.$ $F$ ) appearing in (2.25) induced by the zeroth-order deformation (2.37). Both kinds of modifications can be combined in a single expression of the form

$$
\delta\left(g_{m \bar{n}}^{(0)}+\alpha g_{m \bar{n}}^{(1)}\right)=-i \delta v^{i}\left(\omega_{i m \bar{m}}+\alpha \nabla_{m}^{(0)} \nabla_{\bar{n}}^{(0)} \lambda_{i}\right)+\mathcal{O}\left(\alpha^{2}\right)
$$

for suitable functions $\lambda_{i}$. Similar results were found in [25, 26]. Crucially, these functions drop out from the dimensional reduction discussed in the next section. As a consequence, we do not need to discuss their specific form, and moreover it seems that they do not have any physical significance. Let us point out that the deformation (2.37) of the zeroth-order metric induces also a modification of the quantity $Q$ entering the order $\alpha$ expressions for the Weyl rescaling function $\Phi^{(1)}$ and the dilaton correction $\phi^{(1)}$. Since we are working up to quadratic order in fluctuations, we can consider a truncated Taylor series expansion for $Q$ of the form

$$
Q\left[g^{(0)}+\delta g^{(0)}\right]=Q+Q_{i} \delta v^{i}+\frac{1}{2} Q_{i j} \delta v^{i} \delta v^{j} .
$$

In order to compute the $4 \mathrm{~d}$ effective action we treat the fluctuation parameters $\delta v^{i}$ as arbitrary functions of the external coordinates. In a similar way, we promote the $\mathcal{O}\left(\alpha^{0}\right)$ dilaton profile $\phi_{0}$ from a constant to an arbitrary function of external spacetime. In summary, the Ansatz utilized in the dimensional reduction takes the form

$$
\begin{aligned}
d s_{10}^{2}= & \exp \left\{-192 \alpha e^{-\frac{3}{2} \phi(x)}\left(Q+Q_{i} \delta v^{i}+\frac{1}{2} Q_{i j} \delta v^{i} \delta v^{j}\right)\right\} \times \\
& \times\left[g_{\mu \nu} d x^{\mu} d x^{\nu}+2\left(g_{m \bar{n}}^{(0)}+\alpha g_{m \bar{n}}^{(1)}-i \delta v^{i}\left(\omega_{i m \bar{n}}+\alpha \nabla_{m}^{(0)} \nabla_{\bar{n}}^{(0)} \lambda_{i}\right)\right) d z^{m} d \bar{z}^{\bar{n}}\right] \\
& +\mathcal{O}\left(\alpha^{2}\right)+\mathcal{O}\left(\delta v^{3}\right), \\
\hat{\phi}(x, y)= & \phi(x)+1152 \tilde{c}_{1} \alpha e^{-\frac{3}{2} \phi(x)}\left(Q+Q_{i} \delta v^{i}+\frac{1}{2} Q_{i j} \delta v^{i} \delta v^{j}\right)+\mathcal{O}\left(\alpha^{2}\right)+\mathcal{O}\left(\delta v^{3}\right) .
\end{aligned}
$$

Note that we have replaced the external Minkowski metric with an arbitrary metric $g_{\mu \nu}(x)$. We will drop the explicit dependence of the spacetime coordinates, writing $\phi$ for $\phi(x)$, for notational simplicity in the following.

\section{$2.3 \quad$ Reduction results}

This section is devoted to the discussion of the results of the dimensional reduction of the various terms in (2.9) according to the Ansatz (2.41). In section 2.3.1 we present the outcome of the computation and we address the problem of uplifting it from small fluctuations $\delta v^{i}$ to a full, non-linear dependence on the Kähler structure moduli. Section 2.3.2 is then devoted to the Weyl rescaling that casts the $4 \mathrm{~d}$ Einstein-Hilbert term into canonical form. 


\subsubsection{Results and uplift}

To begin with, the reduction of the Einstein-Hilbert term to four dimensions yields

$$
\int_{\mathcal{M}_{10}} R *_{10} 1 \longrightarrow \int_{\mathcal{M}_{4}}\left(\Omega R+P_{i j} \nabla_{\mu} \delta v^{i} \nabla^{\mu} \delta v^{j}+P_{i} \nabla_{\mu} \delta v^{i} \nabla^{\mu} \phi\right) *_{4} 1
$$

with

$$
\begin{aligned}
\Omega & =\int_{Y_{3}}\left[1-i \delta v^{i} \omega_{i m}{ }^{m}+\frac{1}{2} \delta v^{i} \delta v^{j}\left(\omega_{i m \bar{n}} \omega_{j}{ }^{\bar{n} m}-\omega_{i m}{ }^{m} \omega_{j n}{ }^{n}\right)-384 \cdot 2 \alpha e^{-\frac{3}{2} \phi_{0}} Q\right] *_{6}^{(0)} 1, \\
P_{i j} & =\int_{Y_{3}}\left[\frac{1}{2} \omega_{i m \bar{n}} \omega_{j} \bar{n} m-\omega_{i m}{ }^{m} \omega_{j n}{ }^{n}-384 \cdot 2 \alpha e^{-\frac{3}{2} \phi_{0}} Q\left(\frac{1}{2} \omega_{i m \bar{n}} \omega_{j}{ }^{\bar{n} m}+\omega_{i m}{ }^{m} \omega_{j n}{ }^{n}\right)\right] *_{6}^{(0)} 1 \\
P_{i} & =\int_{Y_{3}}-384 \cdot 6 i \alpha e^{-\frac{3}{2} \phi} \omega_{i m}{ }^{m} Q *_{6}^{(0)} 1 .
\end{aligned}
$$

The terms proportional to $\alpha$ originate from the $10 \mathrm{~d}$ Weyl rescaling factor in the backreacted metric Ansatz (2.41). Let us point out that terms involving other quantities such as $g_{m \bar{n}}^{(1)}$ and $\lambda_{i}$ drop out of the final result because their contributions can be organized into total derivatives in the internal space. It is also crucial to make use of the fact that the fluctuation of $c_{3}$ under a Kähler deformation is an exact six-form, in accordance with $c_{3}$ being a characteristic class. ${ }^{4}$

Next we consider the reduction of the dilaton kinetic terms. Note that $\nabla_{\mu} \phi$ can be effectively considered to be a fluctuation of the same order as $\delta v^{i}$, and as a result terms of the schematic form $\delta v \nabla \phi \nabla \phi$ or $\delta v \nabla \delta v \nabla \phi$ have to be neglected. To linear order in fluctuations we have

$$
\nabla_{\mu} \phi=\nabla_{\mu} \phi\left[1-1728 \alpha \tilde{c}_{1} e^{-\frac{3}{2} \phi} Q\right]+1154 \alpha \tilde{c}_{1} e^{-\frac{3}{2} \phi} Q_{i} \nabla_{\mu} \delta v^{i} .
$$

Combining this observation with the effect of the Weyl rescaling factor we obtain

$$
\int_{\mathcal{M}_{10}}-\frac{1}{2} \nabla_{M} \hat{\phi} \nabla^{M} \hat{\phi} *_{10} 1 \longrightarrow \int_{\mathcal{M}_{4}}\left[U \nabla_{\mu} \phi \nabla^{\mu} \phi+U_{i} \nabla_{\mu} \phi \nabla^{\mu} \delta v^{i}\right] *_{4} 1
$$

with

$$
U=-\frac{1}{2}\left(2 \pi \alpha^{\prime}\right)^{3} \mathcal{V}^{(0)}+384 \alpha\left(1+\frac{9}{2} \tilde{c}_{1}\right) e^{-\frac{3}{2} \phi}(2 \pi)^{3} \chi, \quad U_{i}=-384 \alpha \cdot 3 i \tilde{c}_{1} \omega_{i m}^{m}(2 \pi)^{3} \chi,
$$

where $\mathcal{V}^{(0)}$ denotes the volume of $Y_{3}$ in units of $\sqrt{2 \pi \alpha^{\prime}}$ computed with the zeroth-order metric $g_{m \bar{n}}^{(0)}$ and we used (2.21) and the footnote for the integrals of $Q, Q_{i}$. We have also recalled that $\omega_{i m}{ }^{m}$ is constant on the threefold.

\footnotetext{
${ }^{4}$ This observation allows us to derive useful identities involving the variation of the zero-form $Q$. Recalling $Q=(2 \pi)^{3} *_{6}^{(0)} c_{3}$ and taking into account the variation of the metric implicit in the Hodge star, one can show that

$$
\int_{Y_{3}}\left[Q_{i}-i \omega_{i m}^{m} Q\right] *_{6}^{(0)} 1=0, \quad \int_{Y_{3}}\left[Q_{i j}+\left(\omega_{i m \bar{n}} \omega_{j}^{\bar{n} m}+\omega_{i m}{ }^{m} \omega_{j n}{ }^{n}\right) Q\right] *_{6}^{(0)} 1=0,
$$

where the quantities $Q_{i}, Q_{i j}$ were introduced in (2.40).
} 
We can now record the results of the reduction of the higher-derivative terms. First of all,

$$
\int_{\mathcal{M}_{10}} e^{-\frac{3}{2} \phi}\left(t_{8} t_{8}+\frac{1}{8} \epsilon_{10} \epsilon_{10}\right) R^{4} *_{10} 1 \longrightarrow \int_{\mathcal{M}_{4}}\left[-384 \cdot 2(2 \pi)^{3} e^{-\frac{3}{2} \phi} \chi R+\tilde{P}_{i j} \nabla_{\mu} \delta v^{i} \nabla^{\mu} \delta v^{j}\right] *_{4} 1,
$$

where

$$
\tilde{P}_{i j}=384(2 \pi)^{3} e^{-\frac{3}{2} \phi} \int_{Y_{3}} \omega_{i m \bar{n}} \omega_{j}^{\bar{n} m} c_{3} .
$$

Let us remind the reader that the internal six-form $c_{3}$ is the third Chern form defined in appendix A and was related to $Q$ in (2.21). Secondly, the reduction of the higher-derivative dilaton couplings in (2.9) yields

$$
\begin{aligned}
& \int_{\mathcal{M}_{10}} e^{-\frac{3}{2} \phi}\left(\tilde{c}_{1} \nabla^{M} \nabla_{M} \hat{\phi} f_{1}+\tilde{c}_{2} \nabla_{M} \hat{\phi} \nabla_{N} \hat{\phi} f_{2}^{M N}+\tilde{c}_{3} \nabla^{M} \hat{\phi} \nabla_{M} \hat{\phi} f_{3}\right) \\
& \longrightarrow 384(2 \pi)^{3} \int_{\mathcal{M}_{4}} e^{-\frac{3}{2} \phi}\left[-\frac{3}{2}\left(3 \tilde{c}_{1}+2 \tilde{c}_{2}+4 \tilde{c}_{3}\right) \chi \nabla_{\mu} \phi \nabla^{\mu} \phi+3 i \tilde{c}_{1} \omega_{i m}{ }^{m} \chi \nabla_{\mu} \phi \nabla^{\mu} \delta v^{i}\right] *_{4} 1 .
\end{aligned}
$$

As mentioned above, in the process of dimensional reduction we have implicitly chosen a reference point in the complex structure and Kähler moduli spaces of the threefold $Y_{3}$ and we have only activated Kähler fluctuations $\delta v^{i}$, retaining terms up to quadratic order. Our next task is to infer the form taken by the couplings written above when the fluctuations $\delta v^{i}$ are promoted to a full dependence on the moduli space of $Y_{3}$. At two-derivative level this is a standard procedure that we briefly review in order to set up our notation.

Recall that the $(1,1)$ forms $\omega_{i m \bar{n}}, i=1, \ldots, h^{1,1}$ are the harmonic representatives of the cohomology classes dual to an integral basis $D_{i}$ of the homology $H_{4}\left(Y_{3}, \mathbb{Z}\right)$. The threefold intersection numbers are denoted

$$
\mathcal{K}_{i j k}=D_{i} \cdot D_{j} \cdot D_{k}=\left(2 \pi \alpha^{\prime}\right)^{-3} \int_{Y_{3}} \omega_{i} \wedge \omega_{j} \wedge \omega_{k}
$$

We have inserted the appropriate power of $2 \pi \alpha^{\prime}$ in order to make the intersection numbers $\mathcal{K}_{i j k}$ dimensionless. Indeed, the components $\omega_{i m \bar{n}}$ are dimensionless, so that the $(1,1)$-forms $\omega_{i}=\omega_{i m \bar{n}} d z^{m} \wedge d \bar{z}^{\bar{n}}$ have mass dimension -2 .

The Kähler class of the zeroth-order metric at the reference point in moduli space can be expanded onto the basis of forms $\omega_{i}$ as

$$
J^{(0)}=v^{(0) i} \omega_{i},
$$

where $v^{(0) i}$ are taken to be dimensionless. We also define the quantities

$$
\mathcal{K}_{i j}^{(0)}=\mathcal{K}_{i j k} v^{(0) k}, \quad \mathcal{K}_{i}^{(0)}=\frac{1}{2} \mathcal{K}_{i j k} v^{(0) j} v^{(0) k}, \quad \mathcal{V}^{(0)}=\frac{1}{6} \mathcal{K}_{j i k} v^{(0) i} v^{(0) j} v^{(0) k}
$$

The quantity $\mathcal{V}^{(0)}$ is the volume of the threefold in units of $\sqrt{2 \pi \alpha^{\prime}}$, so that we can write

$$
\int_{Y_{3}} *_{6}^{(0)} 1=\left(2 \pi \alpha^{\prime}\right)^{3} \mathcal{V}^{(0)} .
$$


Let us record the useful identities

$$
\omega_{i m}{ }^{m}=i \frac{\mathcal{K}_{i}^{(0)}}{\mathcal{V}^{(0)}}, \quad \omega_{i m \bar{n}} \omega_{j}{ }^{\bar{n} m} *_{6}^{(0)} 1=\omega_{i} \wedge \omega_{j} \wedge J-\frac{\mathcal{K}_{i}^{(0)} \mathcal{K}_{j}^{(0)}}{\mathcal{V}^{(0) 2}} *_{6}^{(0)} 1,
$$

and stress that harmonicity of $\omega_{i}$ guarantees that $\omega_{i m}{ }^{m}$ is covariantly constant on $Y_{3}$. Using these identities we can immediately compute the $\mathcal{O}\left(\alpha^{0}\right)$ part of $P_{i j}$ defined in (2.45),

$$
P_{i j}=\left(2 \pi \alpha^{\prime}\right)^{3}\left[\frac{1}{2} \mathcal{K}_{i j}^{(0)}+\frac{1}{2} \frac{\mathcal{K}_{i}^{(0)} \mathcal{K}_{j}^{(0)}}{\mathcal{V}^{(0)}}\right]+\mathcal{O}(\alpha) .
$$

This expression is initially understood to be evaluated at the reference point in Kähler moduli space, but given its topological nature it uplifts naturally to the full moduli space dependence. This is achieved simply by replacing $v^{(0) i}$ with an arbitrary $v^{i}$. The quantities defined in (2.55), (2.56) in terms of $v^{(0) i}$ are promoted to $v$-dependent quantities denoted $\mathcal{V}, \mathcal{K}_{i}, \mathcal{K}_{i j}$ without the ${ }^{(0)}$ superscript. In a similar way, the quantity $\Omega^{\prime}$ naturally uplifts to the full $v$-dependent threefold volume form, which integrates to the full volume.

The uplift of higher-derivative couplings is considerably less under control in general, due to the fact that corrections are expected to lift some of the naïve flat directions of moduli space, making the identification of the correct massless modes a hard problem. Nonetheless, we can recast the reduction results of the higher-derivative terms under consideration in a simple form. Exploiting the fact that $\omega_{i m}{ }^{m}$ is covariantly constant we can immediately perform the uplift of integrals of the form

$$
\int_{Y_{3}} \omega_{i m}{ }^{m} c_{3} \longrightarrow i \chi \frac{\mathcal{K}_{i}}{\mathcal{V}}, \quad \int_{Y_{3}} \omega_{i m}{ }^{m} \omega_{j n}{ }^{n} c_{3} \longrightarrow-\chi \frac{\mathcal{K}_{i} \mathcal{K}_{j}}{\mathcal{V}^{2}}
$$

A similar manipulation for the integral

$$
\int_{Y_{3}} \omega_{i m \bar{n}} \omega_{j}^{\bar{n} m} c_{3}
$$

is not straightforward, since the non-harmonic part $F$ of $c_{3}$ introduced in (2.23) poses an obstruction to the factorization of this expression. In the present context, however, the overall coefficient of this term in the dimensional reduction is zero and we do not have to address this complication.

Let us introduce the field-dependent, dimensionless quantity

$$
\tilde{\chi}=384(2 \pi)^{3} \frac{\alpha}{\left(2 \pi \alpha^{\prime}\right)^{3} \mathcal{V}} e^{-\frac{3}{2} \phi} \chi=\frac{1}{16} \zeta(3) \frac{e^{-\frac{3}{2} \phi}}{\mathcal{V}} \chi
$$

In what follows it plays the role of the effective expansion parameter for the problem at hand. Combining all contributions after uplift the $4 \mathrm{~d}$ effective action takes the form

$$
\begin{aligned}
S_{4 \mathrm{~d}}= & \frac{1}{(2 \pi)^{4} \alpha^{\prime}} \int_{\mathcal{M}_{4}}\left\{\mathcal{V}\left(1+b_{1} \tilde{\chi}\right) R+\left[\left(\frac{1}{2}+b_{2} \tilde{\chi}\right) \mathcal{K}_{i j}+\left(\frac{1}{2}+b_{3} \tilde{\chi}\right) \frac{\mathcal{K}_{i} \mathcal{K}_{j}}{\mathcal{V}}\right] \nabla_{\mu} v^{i} \nabla^{\mu} v^{j}\right. \\
& \left.+\mathcal{V}\left(-\frac{1}{2}+b_{4} \tilde{\chi}\right) \nabla_{\mu} \phi \nabla^{\mu} \phi+b_{5} \tilde{\chi} \mathcal{K}_{i} \nabla_{\mu} \phi \nabla^{\mu} v^{i}\right\} *_{4} 1
\end{aligned}
$$


In this expression we have promoted the fluctuations $\delta v^{i}$ to full $4 \mathrm{~d}$ fields $v^{i}$. For notational convenience we have introduced the numerical coefficients

$$
\begin{array}{llrl}
b_{1}=-4, & b_{2}=0, & b_{3}=2 \\
b_{4}=1-3 \tilde{c}_{2}-6 \tilde{c}_{3}, & b_{5}=6 . &
\end{array}
$$

\subsubsection{Weyl rescaling}

Our next task is performing a Weyl rescaling of the external metric in (2.63) in order to bring the $4 \mathrm{~d}$ Einstein-Hilbert term into canonical form. Let us record the identities

$$
g_{\mu \nu}=e^{2 \Lambda} \tilde{g}_{\mu \nu}, \quad R=e^{-2 \Lambda}\left[\tilde{R}-6 \tilde{\nabla}_{\mu} \Lambda \tilde{\nabla}^{\mu} \Lambda-6 \tilde{\nabla}_{\mu} \tilde{\nabla}^{\mu} \Lambda\right] .
$$

In our case, we set

$$
\Lambda=-\frac{1}{2} \log \left[\mathcal{V}\left(1+b_{1} \tilde{\chi}\right)\right] .
$$

Dropping the tilde on the new metric, the Weyl-rescaled $4 \mathrm{~d}$ action can be written in the form

$$
S_{4 \mathrm{~d}}=\frac{1}{(2 \pi)^{4} \alpha^{\prime}} \int_{\mathcal{M}_{4}}\left[R+\mathcal{G}_{v^{i} v^{j}} \nabla_{\mu} v^{i} \nabla^{\mu} v^{j}+\mathcal{G}_{\phi \phi} \nabla_{\mu} \phi \nabla^{\mu} \phi+2 \mathcal{G}_{\phi v^{i}} \nabla_{\mu} \phi \nabla^{\mu} v^{i}\right] *_{4} 1
$$

where

$$
\begin{aligned}
\mathcal{G}_{v^{i} v^{j}} & =-\frac{1}{\mathcal{V}^{2}}\left[1+\tilde{\chi}\left(-b_{3}-\frac{5}{2} b_{1}\right)\right] \mathcal{K}_{i} \mathcal{K}_{j}+\frac{1}{2 \mathcal{V}}\left[1+\tilde{\chi}\left(2 b_{2}-b_{1}\right)\right] \mathcal{K}_{i j}, \\
\mathcal{G}_{\phi \phi} & =-\frac{1}{2}\left[1+\tilde{\chi}\left(-b_{1}-2 b_{4}\right)\right], \\
\mathcal{G}_{\phi v^{i}} & =\left(\frac{9}{4} b_{1}+\frac{1}{2} b_{5}\right) \tilde{\chi} \frac{\mathcal{K}_{i}}{\mathcal{V}} .
\end{aligned}
$$

This form of the reduction result is a convenient starting point for the discussion of the $4 \mathrm{~d}$ $\mathcal{N}=2$ and $\mathcal{N}=1$ theories in the following sections.

\section{Correction to the $\mathcal{N}=2$ prepotential}

In this section we analyze the result of the dimensional reduction from the point of view of the $4 \mathrm{~d}, \mathcal{N}=2$ effective theory obtained without including branes or performing any orientifold projection. We show that our findings are compatible with the known results about the correction to the geometry of the $\mathcal{N}=2$ hypermultiplet scalar manifold induced by a perturbative $\alpha^{\prime}$ correction to the Kähler moduli space prepotential $[21,40,43]$.

\subsection{Translation into $\mathcal{N}=2$ field variables}

Let us recall some well-known facts about the $4 \mathrm{~d}, \mathcal{N}=2$ effective theory arising from compactification of type IIB superstring on a Calabi-Yau threefold [20, 22, 44, 45]. The complex structure moduli of the threefold fit into $n_{V}=h^{1,2}$ vector multiplets. Their scalar manifold is special Kähler and its geometry is tree-level exact both in $g_{s}$ and $\alpha^{\prime}$. The Kähler structure moduli, the dilaton, the axion, as well as the scalars coming from the expansion 
of $B_{2}, C_{2}, C_{4}$ onto internal even harmonic forms, all fit into $n_{H}=h^{1,1}+1$ hypermultiplets. Their scalar manifold $\mathcal{M}_{\mathrm{Q}}$ is quaternionic of real dimension $4\left(h^{1,1}+1\right)$.

The structure of the metric on $\mathcal{M}_{\mathrm{Q}}$ is most conveniently analyzed using the string frame Kähler moduli $v_{\mathrm{s}}^{i}$ and the $4 \mathrm{~d}$ dilaton $\phi_{4}$. In terms of these variables the quaternionic metric on $\mathcal{M}_{\mathrm{Q}}$ can be written schematically as $[20,44]$

$$
d s^{2}\left(\mathcal{M}_{\mathrm{Q}}\right)=\left(d \phi_{4}\right)^{2}+G_{i \bar{\jmath}}\left(w_{\mathrm{s}}, \bar{w}_{\mathrm{s}}\right) d w^{i} d \bar{w}^{\bar{\jmath}}+\mathcal{G}_{x y}\left(w_{\mathrm{s}}, \bar{w}_{\mathrm{s}}, \phi_{4}, q\right) d q^{x} d q^{y} .
$$

In this expression $w_{\mathrm{s}}^{i}=u_{\mathrm{s}}^{i}+i v_{\mathrm{s}}^{i}$ are the complexified Kähler moduli arising from expansion of $B_{2}+i J_{\mathrm{s}}$ onto the basis $\omega_{i}$ of harmonic $(1,1)$-forms. The metric $G_{i \bar{\jmath}}$ is the special Kähler metric on the Kähler moduli space $\mathcal{M}_{\mathrm{K}}$ of the threefold. The fields $q^{x}$ denote collectively all real scalars in $\mathcal{M}_{\mathrm{Q}}$ different from $\phi_{4}, u_{\mathrm{s}}^{i}, v_{\mathrm{s}}^{i}$. The metric components $\mathcal{G}_{x y}$ are entirely determined by the special Kähler metric on $\mathcal{M}_{\mathrm{K}}$. For this reason, the quaternionic metric on $\mathcal{M}_{\mathrm{Q}}$ is sometimes referred to as special quaternionic [20,44]. Let us stress that the structure (3.1) of the metric on $\mathcal{M}_{\mathrm{Q}}$ is expected to hold not only at tree level, but also including $\alpha^{\prime}$ corrections, thanks to mirror symmetry considerations, reviewed for instance in $[46,47]$.

In our analysis all the fields $q^{x}$ as well as the axions $u_{\mathrm{s}}^{i}$ are effectively frozen to zero. Our next task is therefore to connect the string frame Kähler moduli $v_{\mathrm{s}}^{i}$ and the $4 \mathrm{~d}$ dilaton $\phi_{4}$ to the Einstein frame Kähler moduli $v^{i}$ and the 10d dilaton $\phi$. At two-derivative level we have the relations

$$
e^{-2 \phi_{4}}=e^{-\frac{1}{2} \phi} \mathcal{V}, \quad v_{\mathrm{s}}^{i}=e^{\frac{1}{2} \phi} v^{i} .
$$

Let us remind the reader that in our conventions $\mathcal{V}$ is the volume of the threefold in units of $\sqrt{2 \pi \alpha^{\prime}}$. In order to take into account the effect of higher-derivative corrections we deform these relations into

$$
e^{-2 \phi_{4}}=e^{-\frac{1}{2} \phi} \mathcal{V}\left(1+\Upsilon_{1} \tilde{\chi}\right), \quad v_{\mathrm{s}}^{i}=e^{\frac{1}{2} \phi} v^{i}\left(1+\Upsilon_{2} \tilde{\chi}\right)
$$

where $\Upsilon_{1}, \Upsilon_{2}$ are constants that will be fixed momentarily.

We can now invert (3.3) to leading order in $\alpha^{\prime 3}$ to obtain $v^{i}, \phi$ in terms of $v_{\mathrm{s}}^{i}, \phi_{4}$, and plug the resulting expressions into the $4 \mathrm{~d}$ effective action (2.68). The result is conveniently expressed in terms of the quantities

$$
\mathcal{V}_{\mathrm{s}}=\frac{1}{6} \mathcal{K}_{i j k} v_{\mathrm{s}}^{i} v_{\mathrm{s}}^{j} v_{\mathrm{s}}^{k}, \quad \mathcal{K}_{i}^{\mathrm{s}}=\frac{1}{2} \mathcal{K}_{i j k} v_{\mathrm{s}}^{j} v_{\mathrm{s}}^{k}, \quad \mathcal{K}_{i j}^{\mathrm{s}}=\mathcal{K}_{i j k} v_{\mathrm{s}}^{k}
$$

Furthermore, it is convenient to introduce a new field-dependent dimensionless quantity $\tilde{\chi}_{\mathrm{s}}$, which is the string-frame analog of $\tilde{\chi}$ and is given by

$$
\tilde{\chi}_{\mathrm{s}}=\frac{1}{16} \zeta(3) \frac{1}{\mathcal{V}_{\mathrm{s}}} \chi .
$$

With this notation, the $4 \mathrm{~d}$ effective action can be written as

$$
\begin{aligned}
S_{4 \mathrm{~d}}= & \frac{1}{(2 \pi)^{4} \alpha^{\prime}} \int_{\mathcal{M}_{4}}\left\{R+\left[\frac{1}{2 \mathcal{V}_{\mathrm{s}}}\left(1+a_{1} \tilde{\chi}_{\mathrm{s}}\right) \mathcal{K}_{i j}^{\mathrm{s}}-\frac{1}{2 \mathcal{V}_{\mathrm{s}}}\left(1+a_{2} \tilde{\chi}_{\mathrm{s}}\right) \frac{\mathcal{K}_{i}^{\mathrm{s}} \mathcal{K}_{j}^{\mathrm{s}}}{\mathcal{V}_{\mathrm{s}}}\right] \nabla_{\mu} v_{\mathrm{s}}^{i} \nabla^{\mu} v_{\mathrm{s}}^{j}\right. \\
& \left.-2\left(1+a_{3} \tilde{\chi}_{\mathrm{s}}\right) \nabla_{\mu} \phi_{4} \nabla^{\mu} \phi_{4}+a_{4} \tilde{\chi}_{\mathrm{s}} \frac{\mathcal{K}_{i}^{\mathrm{s}}}{\mathcal{V}_{\mathrm{s}}} \nabla_{\mu} \phi_{4} \nabla^{\mu} v_{\mathrm{s}}^{i}\right\} *_{4} 1
\end{aligned}
$$


where the numerical coefficients $a$ are given in terms of the $b$ coefficients introduced in $(2.63)$ by

$$
\begin{aligned}
a_{1} & =2 b_{2}-b_{1}, & a_{2} & =-\frac{37}{16} b_{1}+\frac{5}{4} b_{2}-\frac{1}{8} b_{3}-\frac{1}{2} b_{4}-\frac{1}{4} b_{5}+2 \Upsilon_{2}, \\
a_{3} & =\frac{11}{16} b_{1}-\frac{3}{4} b_{2}-\frac{9}{8} b_{3}-\frac{1}{2} b_{4}+\frac{3}{4} b_{5}, & a_{4} & =-\frac{27}{8} b_{1}-\frac{1}{2} b_{2}-\frac{3}{4} b_{3}+b_{4}-\frac{1}{2} b_{5}+2 \Upsilon_{1} .
\end{aligned}
$$

If we plug in the values of the $b$ coefficients given in (2.64), we find

$$
\begin{array}{rlrl}
a_{1} & =4, & a_{2} & =7+\frac{3}{2} \tilde{c}_{2}+3 \tilde{c}_{3}+2 \Upsilon_{2}, \\
a_{3} & =-1+\frac{3}{2} \tilde{c}_{2}+3 \tilde{c}_{3}, & a_{4}=13-3 \tilde{c}_{2}-6 \tilde{c}_{3}+2 \Upsilon_{1} .
\end{array}
$$

We are now in a position to discuss the correction to the $\mathcal{N}=2$ prepotential.

\subsection{Implications for $\mathcal{N}=2$ prepotential}

As noted above, the form (3.1) of the quaternionic metric on $\mathcal{M}_{\mathrm{Q}}$ should be preserved by $\alpha^{\prime}$ corrections. In particular, cross terms between $\phi_{4}$ and $v_{\mathrm{s}}^{i}$ are not allowed, and also $v_{\mathrm{s}}^{i}$-dependent corrections to the $\left(d \phi_{4}\right)^{2}$ terms are forbidden. These considerations lead us to impose $a_{3}=0, a_{4}=0$. As a result we can fix the value of $\Upsilon_{1}$ and derive a linear constraint on the dilaton coupling coefficients $\tilde{c}_{2}, \tilde{c}_{3}$,

$$
\Upsilon_{1}=-4, \quad 3 \tilde{c}_{2}+6 \tilde{c}_{3}-2=0 .
$$

We now aim at demonstrating that the effective action (3.6) can be written as

$S=\frac{1}{(2 \pi)^{4} \alpha^{\prime}} \int_{\mathcal{M}_{4}}\left\{R-2 \nabla_{\mu} \phi_{4} \nabla^{\mu} \phi_{4}-2 G_{i \bar{\jmath}} \nabla_{\mu} v_{\mathrm{s}}^{i} \nabla^{\mu} v_{\mathrm{s}}^{j}\right\} *_{4} 1, \quad G_{i \bar{\jmath}}=\partial_{w_{\mathrm{s}}^{i}} \partial_{\bar{w}_{\mathrm{s}}^{\bar{s}}} K^{\mathcal{N}=2}\left(w_{\mathrm{s}}, \bar{w}_{\mathrm{s}}\right)$,

where we remind the reader that $w_{\mathrm{s}}^{i}=u_{\mathrm{s}}^{i}+i v_{\mathrm{s}}^{i}$ are the complexified Kähler moduli, and where the Kähler potential $K^{\mathcal{N}=2}\left(w_{\mathrm{s}}, \bar{w}_{\mathrm{s}}\right)$ can be derived from a holomorphic prepotential.

In order to set our notation, it is useful to review the relation between the Käher potential and the prepotential. The geometry of the $h^{1,1}$ complex-dimensional Kähler moduli space is conveniently described by $h^{1,1}+1$ complex projective coordinates $X^{I}=$ $\left(X^{0}, X^{i}\right)$ related to the complex coordinates $w_{\mathrm{s}}^{i}$ by

$$
w_{\mathrm{s}}^{i}=\frac{X^{i}}{X^{0}} .
$$

The geometry of $\mathcal{M}_{\mathrm{K}}$ is encoded in a holomorphic prepotential $F(X)$, which is a homogeneous function of $X^{I}$ of degree 2. The Kähler potential $K^{\mathcal{N}=2}\left(w_{\mathrm{s}}, \bar{w}_{\mathrm{s}}\right)$ for the metric on $\mathcal{M}_{\mathrm{K}}$ is then extracted from $F$ via

$$
e^{-K^{\mathcal{N}=2}\left(w_{\mathrm{s}}, \bar{w}_{\mathrm{s}}\right)}=-i\left(X^{I} \bar{F}_{I}-F_{I} \bar{X}^{I}\right), \quad F_{I}=\partial_{X^{I}} F(X) .
$$

Mirror symmetry considerations [21, 46, 47] ensure that the prepotential $F(X)$ takes the form

$$
F(X)=\frac{1}{X^{0}} \frac{1}{6} \mathcal{K}_{i j k} X^{i} X^{j} X^{k}+i \lambda\left(X^{0}\right)^{2}+\ldots
$$


where the first term is the classical prepotential, the second is a perturbative $\alpha^{\prime}$ effect, and the ellipsis stands for terms that are exponentially suppressed in the large volume limit and originate from worldsheet instantons. The $\mathcal{N}=2$ Kähler potential derived from (3.13) using (3.12) is, up to instanton corrections and an immaterial constant,

$$
K^{\mathcal{N}=2}=-\log \left[\mathcal{V}_{\mathrm{s}}+\frac{1}{2} \lambda\right]
$$

We can finally relate this to our dimensional reduction by comparing the metric computed from (3.14) with the metric in (3.6). We find that, in order for the match to be possible, we have to impose

$$
\Upsilon_{2}=0
$$

The constant $\lambda$ is then fixed to be

$$
\lambda=-\frac{1}{2} \zeta(3) \chi
$$

so that the $\mathcal{N}=2$ Kähler potential reads

$$
K^{\mathcal{N}=2}=-\log \left[\frac{\mathcal{V}_{\mathrm{s}}}{\left(2 \pi \alpha^{\prime}\right)^{3}}-\frac{1}{4} \zeta(3) \chi\right] .
$$

In this last expression we have reinstated all factors of $\alpha^{\prime}$ and $\mathcal{V}_{\mathrm{s}}$ denotes the dimensionful volume of the threefold. Let us close this section with a comparison between our findings and the analogous quantities in BBHL [6]. Using equations (3.11), (3.12), (3.13) and the comment before (3.15) in that paper, we infer that their $\mathcal{N}=2$ Kähler potential in our notation coincides exactly with (3.17).

\section{$4 \mathcal{N}=1$ Kähler coordinates and Kähler potential}

In this section we analyze the results of the dimensional reduction and identify the Kähler coordinates and Kähler potential in the $4 \mathrm{~d}, \mathcal{N}=1$ effective action. We briefly comment on our findings.

\subsection{Correction to the Kähler potential and coordinates}

Upon dimensional reduction on a Calabi-Yau threefold, the two-derivative action of type IIB supergravity yields a $4 \mathrm{~d}$ effective action with $\mathcal{N}=2$ supersymmetry. If orientifold planes are included in the setup, the $4 \mathrm{~d}$ spectrum is suitably projected and one obtains an effective action with $4 \mathrm{~d}, \mathcal{N}=1$ supersymmetry [30-32]. For definiteness, we consider here the projection relevant to the case with $03 / O 7$-planes. The dilaton $\phi$ and the Kähler moduli $v^{i}$ fit into $4 \mathrm{~d}, \mathcal{N}=1$ chiral multiplets whose scalar components are of the form

$$
\begin{aligned}
\tau_{0} & =C_{0}+i e^{-\phi}, \\
T_{i} & =\rho_{i}+i \mathcal{K}_{i}+\zeta_{i} .
\end{aligned}
$$

Several remarks are in order. We used the symbol $\tau_{0}$ to denote the $4 \mathrm{~d}$ axio-dilaton. Its real part $C_{0}$ is the straightforward dimensional reduction of the fluctuations of the $10 \mathrm{~d}$ axion 
around its zero background. Its imaginary part is built with $\phi$ which is not directly identified with the $10 \mathrm{~d}$ dilaton because of the backreaction term $\phi^{(1)}$ discussed in section 2.2.1. This discrepancy has consequences for the transformation property of $4 \mathrm{~d}$ fields under the $10 \mathrm{~d} \operatorname{SL}(2, \mathbb{Z})$ symmetry, as discussed in [6]. In the expression for $T_{i}$, the scalars $\rho_{i}$ come from the expansion of the RR four-form, and $\zeta_{i}$ denotes a complex quantity built with the scalars coming from the reduction of the NSNS and RR two-forms. Since we are freezing all such scalars to zero, the quantity $\zeta_{i}$ vanishes. Let us point out that the orientifold projection to $\mathcal{N}=1$ supersymmetry is implicit in the range of the index $i$. Indeed, by an abuse of notation, the index $i$ in (4.1) runs only over the elements of $H_{+}^{1,1}\left(Y_{3}\right)$, i.e. the $(1,1)$ forms that are even under the isometric involution of $Y_{3}$ considered in the implementation of the orientifold projection. Let us stress that equations (2.56) still hold after restricting the range of $i$, by virtue of the restrictions imposed on intersection numbers by the orientifold projection, as explained in detail in [30-32].

The kinetic terms of $\tau_{0}$ and $T_{i}$ are governed by a Kähler potential $K$. If we write $T_{0} \equiv \tau_{0}$ and introduce the collective index $I=(0, i)$, the effective action contains the terms

$$
S_{4 \mathrm{~d}} \supset \frac{1}{(2 \pi)^{4} \alpha^{\prime}} \int_{\mathcal{M}_{4}}\left[R+G_{T_{I} \bar{T}_{J}} \nabla_{\mu} T_{I} \nabla^{\mu} \bar{T}_{J}\right] *_{4} 1
$$

where

$$
G_{T_{I} \bar{T}_{J}}=-2 \partial_{T_{I}} \partial_{\bar{T}_{J}} K, \quad K=\phi-2 \log \mathcal{V}
$$

The Kähler potential is understood as a function of $T_{I}, \bar{T}_{J}$ determined implicitly via (4.1).

Upon inclusion of higher-derivative terms in the $10 \mathrm{~d}$ action, the coefficients of the terms in the two-derivative $4 \mathrm{~d}$ effective action are modified, but the form of the action is still expected to obey the constraints deriving from $4 \mathrm{~d}, \mathcal{N}=1$ supersymmetry. In this section we show that our results are consistent with this expectation.

Let us introduce the notation $v^{0} \equiv \phi$, in such a way that $v^{I}=\left(\phi, v^{i}\right)$. The kinetic terms specified by (2.69) can be written compactly as

$$
\mathcal{G}_{v^{I} v^{J}} \nabla_{\mu} v^{I} \nabla^{\mu} v^{J}=\mathcal{G}_{\phi \phi} \nabla_{\mu} \phi \nabla^{\mu} \phi+\mathcal{G}_{v^{i} v^{j}} \nabla_{\mu} v^{i} \nabla^{\mu} v^{j}+2 \mathcal{G}_{v^{i} \phi} \nabla_{\mu} v^{i} \nabla^{\mu} \phi
$$

Recall that $C_{0}, \rho_{i}$ and $\zeta_{i}$ are frozen to zero in our discussion. The problem at hand is the determination of the $\mathcal{O}(\tilde{\chi})$ corrections to $T_{I}$ and $K$ in such a way that the relation

$$
\mathcal{G}_{v^{I} v^{J}} \nabla_{\mu} v^{I} \nabla^{\mu} v^{J}=-2\left(\partial_{T_{I}} \partial_{\bar{T}_{J}} K\right) \nabla_{\mu} T_{I} \nabla^{\mu} \bar{T}_{J}
$$

holds including terms up to order $\tilde{\chi}$.

The first outcome of our analysis is the observation that, regarding the $b$ coefficients in (2.63) as input data, a solution exists only if the following linear constraint is satisfied,

$$
11 b_{1}-12 b_{2}-18 b_{3}-8 b_{4}+12 b_{5}=0 \text {. }
$$

Plugging in the values of the $b$ coefficients given in (2.64), we obtain

$$
3 \tilde{c}_{2}+6 \tilde{c}_{3}-2=0
$$


which is the same constraint on $\tilde{c}_{2}, \tilde{c}_{3}$ found in (3.9) in the analysis of the $\mathcal{N}=2$ case. If this requirement is met, the Kähler coordinates and Kähler potential are given in terms of the $b$ coefficients by

$$
\begin{aligned}
& \tau_{0}=i e^{-\phi}\left[1+\tilde{\chi}\left(-\frac{3}{4} b_{1}+\frac{3}{2} b_{3}-b_{5}\right)\right], \\
& T_{i}=i \mathcal{K}_{i}\left[1+\tilde{\chi}\left(\frac{1}{4} b_{1}+2 b_{2}+\frac{1}{2} b_{3}\right)\right] \\
& K=\phi-2 \log \left[\mathcal{V}\left(1+\tilde{\chi}\left(b_{1}+b_{2}+\frac{3}{2} b_{3}-\frac{1}{2} b_{5}\right)\right)\right] .
\end{aligned}
$$

The actual values taken by these quantities upon substituting the numerical values of the $b$ coefficients are summarized at the end of this section.

An important feature of the zeroth-order Kähler potential is the property of extended no-scale structure [30-32]. This property is tested by computing the following quantity,

$$
\frac{\partial K}{\partial T_{I}}\left(K^{-1}\right)_{I \bar{J}} \frac{\partial K}{\partial \bar{T}_{\bar{J}}}=4\left[1+0 \cdot \tilde{\chi}+\mathcal{O}\left(\tilde{\chi}^{2}\right)\right]
$$

where $\left(K^{-1}\right)_{I \bar{J}}$ denotes the inverse of the matrix $K^{I \bar{J}}=\partial_{T_{I}} \partial_{\bar{T}_{J}} K$. The zeroth order value 4 signals extended no-scale structure [48], and interestingly the leading correction has a vanishing coefficient for any value of the $b$ coefficients. This does not mean that the correction under examination has no physical effect. For instance, in a scenario like KKLT the axio-dilaton and the complex structure moduli are stabilized first at a supersymmetric value by means of the Gukov-Vafa-Witten superpotential [49]. The relevant quantity in the computation of the scalar potential for the Kähler moduli is no longer the quantity in (4.12), but rather the same object with summation restricted to $i, j$ indices only, implicitly evaluated at the fixed value of the axio-dilaton. One finds

$$
\frac{\partial K}{\partial T_{i}}\left(K^{-1}\right)_{i \bar{\jmath}} \frac{\partial K}{\partial \bar{T}_{\bar{\jmath}}}=3\left[1+\left(\frac{1}{2} b_{1}-b_{2}\right) \tilde{\chi}+\mathcal{O}\left(\tilde{\chi}^{2}\right)\right] .
$$

As we can see, the zeroth-order value 3 associated to no-scale structure receives a non-zero correction at order $\tilde{\chi}\left(\frac{1}{2} b_{1}-b_{2}=-2\right)$.

In order to elucidate further the physical effects of the correction to the Kähler potential an alternative formulation can be used, in which the chiral multiplets $T_{i}$ are dualized into linear multiplets $L_{i}$ [30-32]. The dynamics of the system is encoded in a kinetic potential $\tilde{K}$, which is the Legendre transform of the original Kähler potential $K$. Let us adopt the following conventions for the Legendre transform,

$$
L^{i}=-\frac{\partial K}{\partial \operatorname{Im} T_{i}}, \quad \tilde{K}=K+L^{i} \operatorname{Im} T_{i}, \quad \operatorname{Im} T_{i}=\frac{\partial \tilde{K}}{\partial L^{i}} .
$$

A straightforward computation gives, up to terms of order $\tilde{\chi}^{2}$ and higher,

$$
\begin{aligned}
L_{i} & =\frac{v^{i}}{\mathcal{K}}\left[1+\tilde{\chi}\left(-\frac{5}{4} b_{1}-\frac{1}{2} b_{3}\right)\right], \\
L^{i} \operatorname{Im} T_{i} & =3\left[1+\tilde{\chi}\left(-b_{1}+2 b_{2}\right)\right] .
\end{aligned}
$$


In this formulation the hallmark of zeroth-order no-scale structure is the value 3 , which again receives corrections at order $\tilde{\chi}$. As a further check that the $4 \mathrm{~d}$ dynamics is really affected by the corrections under examination we can examine the expression for the kinetic potential $\tilde{K}$ in terms of the fields $L^{i}$,

$$
\tilde{K}=3-\log \operatorname{Im} \tau+\log \left(\frac{1}{6} \mathcal{K}_{i j k} L^{i} L^{j} L^{k}\right)+\frac{1}{16} \zeta(3) \chi\left(-2 b_{1}+4 b_{2}\right)(\operatorname{Im} \tau)^{3 / 2} \sqrt{\frac{1}{6} \mathcal{K}_{i j k} L^{i} L^{j} L^{k}},
$$

which has manifestly a different functional form as compared to the zeroth-order result. Note indeed that $-2 b_{1}+4 b_{2}=8$.

Let us now summarize the expressions for the Kähler coordinates and potential after plugging in the values of the $b$ coefficients given in (2.64). We have

$$
\begin{aligned}
\tau_{0} & =i e^{-\phi}, \\
T_{i} & =i \mathcal{K}_{i}, \\
K & =\phi-2 \log \left[\frac{\mathcal{V}}{\left(2 \pi \alpha^{\prime}\right)^{3}}-\frac{1}{4} \zeta(3) e^{-\frac{3}{2} \phi} \chi\right], \\
L_{i} & =\frac{v^{i}}{\mathcal{V}}\left[1+\frac{1}{4} \frac{\left(2 \pi \alpha^{\prime}\right)^{3}}{\mathcal{V}} \zeta(3) e^{-\frac{3}{2} \phi} \chi\right], \\
L^{i} \operatorname{Im} T_{i} & =3\left[1+\frac{1}{4} \frac{\left(2 \pi \alpha^{\prime}\right)^{3}}{\mathcal{V}} \zeta(3) e^{-\frac{3}{2} \phi} \chi\right] .
\end{aligned}
$$

Clearly these expressions are valid up to $\alpha^{\prime}$ corrections of higher order than the $\alpha^{\prime 3}$ corrections under examination. We have utilized the explicit expression (2.62) for $\tilde{\chi}$ and we have used $\mathcal{V}$ for the dimensionful volume of the threefold, thus reinstating explicitly all factors of $\alpha^{\prime}$ for the convenience of the reader. As we can see, the leading correction to $\tau_{0}$, $T_{i}$ is vanishing. Let us also remark that our finding for the correction to the $\mathcal{N}=1$ Kähler potential is in perfect agreement with the corresponding result in BBHL [6].

\subsection{Some comments}

Some comments about the results of the previous section are in order. We consider the $4 \mathrm{~d}, \mathcal{N}=1$ theory arising from a Calabi-Yau orientifold with $O 7$-planes and D7-branes, but we do not take into account explicitly the backreaction of these extended objects. For instance, the Chern-Simons part of the effective action for a D7-brane wrapping a divisor in the Calabi-Yau threefold contains higher-curvature terms which are essential in the derivation of the contribution of $D 7$-branes to the $D 3$-brane tadpole cancellation condition [35]. From the point of view of the uplift to F-theory, D7-branes and O7-branes are encoded in the Calabi-Yau fourfold geometry, and the metric Ansatz for dimensional reduction to three dimensions is modified by higher-derivative corrections [24-28]. These considerations suggest that $D 7$-branes and $O 7$-branes could play an important role in the context of higher-derivative corrections to the bulk $\mathcal{N}=1$ Kähler potential, which is however beyond the scope of this work and left for future investigation.

It is interesting to observe that our computation shows that the leading correction to the $\mathcal{N}=1$ Kähler coordinates $\tau_{0}, T_{i}$ is vanishing. As far as the $4 \mathrm{~d}$ axio-dilaton $\tau_{0}$ 
is concerned, this result is consistent with the expectations from F-theory, since in the context of F-theory compactification the axio-dilaton is identified with one of the complex structure moduli of the elliptically fibered Calabi-Yau fourfold, and is therefore expected not to receive corrections from the Kähler moduli sector proportional to the Euler number $\chi$ of the base threefold.

\section{Conclusions}

In this paper we revisited the leading $\alpha^{\prime 3}$ perturbative correction to the Kähler potential of a $4 \mathrm{~d}, \mathcal{N}=1$ orientifold compactification of type IIB superstring theory on a Calabi-Yau threefold. We reproduced the result of Becker, Becker, Haack, and Louis (BBHL) [6] by showing that a correction to the Kähler potential emerges, which is proportional to the Euler characteristic of the leading order Calabi-Yau threefold background.

Our derivation is based on an explicit Kaluza-Klein reduction of the type IIB bulk action from ten to four dimensions. The 10d two-derivative action is supplemented by the well-known gravitational correction of the schematic form $\left(t_{8} t_{8}+\frac{1}{8} \epsilon_{10} \epsilon_{10}\right) R^{4}$ [10-19], as well as by related dilaton couplings. Since the latter are not completely known, we parametrized them in terms of three constants $\tilde{c}_{1}, \tilde{c}_{2}, \tilde{c}_{3}$, see (2.8). Supersymmetry considerations in four dimensions allowed us to derive the linear relation (4.8) among these parameters in the $10 \mathrm{~d}$ action, which could be checked against proposals in the literature for the complete axio-dilaton $\alpha^{\prime 3}$ sector.

The background geometry used in the dimensional reduction at two-derivative level must be modified in order to solve the higher-dimensional equations of motion in the presence of the $\alpha^{\prime 3}$ corrections under examination. We presented an explicit solution for this backreacted background in terms of the non-harmonic part of the third Chern form $c_{3}$ of the zeroth order Calabi-Yau threefold. The $\alpha^{\prime}$-corrected internal metric is still Kähler but no longer Ricci-flat. As a result, the manifold is no longer a Calabi-Yau threefold with SU(3) holonomy, but rather an almost Calabi-Yau threefold [23] endowed with an SU(3) structure for which the only non-zero torsion class is $\mathcal{W}_{5}$ in the notation of [41]. We also found that the entire 10d background metric is corrected at order $\alpha^{\prime 3}$ by an overall Weyl factor proportional to the Euler density of the zeroth order Calabi-Yau threefold. This is in analogy with the results of [24-26] in the context of three-dimensional M-theory vacua in the presence of higher-derivative corrections.

The dimensional reduction is performed by allowing for small spacetime dependent fluctuations $\delta v^{i}$ of the Kähler moduli of the threefold, for arbitrary $h^{1,1}$. We have showed that the outcome of the dimensional reduction can be uplifted from infinitesimal variations to a full non-linear dependence on the moduli space. The latter is captured by simple topological expressions proportional to the Euler characteristic of the threefold.

The resulting kinetic terms for the dilaton and the Kähler moduli are consistent with $4 \mathrm{~d}, \mathcal{N}=2$ supersymmetry, once the appropriate $\mathcal{N}=2$ field variables are identified. The prepotential for the special Kähler geometry of the Kähler moduli space is found to be corrected by the expected term proportional to the Euler characteristic of the threefold [43]. 
The kinetic terms in the $4 \mathrm{~d}$ effective action can also be truncated in accordance with the standard O3/O7 orientifold projection, yielding a theory with $\mathcal{N}=1$ supersymmetry. We reproduce the expected BBHL correction to the $\mathcal{N}=1$ Kähler potential proportional to the Euler characteristic of the classical Calabi-Yau threefold. We also computed the leading $\alpha^{\prime}$ corrections to the Kähler coordinates as a function of the threefold Kähler moduli, and found that they are vanishing.

Our investigation can be extended in several directions. It would be desirable to switch on additional type IIB fields, for instance the axion $C_{0}$, and examine their kinetic terms to confirm the structure of the Kähler potential. It would be also interesting to repeat a similar analysis for the complex structure moduli sector, even though no analogous correction to the Kähler potential is expected. Finally, it would be interesting to consider explicitly the effect of both local sources and bulk higher-derivative corrections on the background solution. To pursue these directions further, however, a preliminary investigation of the complete $\alpha^{\prime 3}$-corrected type IIB bulk action would be probably necessary. By a similar token, the detailed knowledge of the $\alpha^{\prime}$-corrected 10d gravitino and dilatino supersymmetry variations would allow a direct check of supersymmetry of the background in the presence of higher-derivative corrections.

\section{Acknowledgments}

We would like to thank Ralph Blumenhagen, Thomas Grimm, Thomas Hahn, Kilian Mayer and Raffaele Savelli for interesting discussions and correspondence. M.W. would like to express his thankfulness to the theoretical high-energy physics groups of the center for the fundamental laws of nature in Harvard, and the Max-Planck institute for physics in Munich, for their hospitality during my visits. This work was supported by the WPI program of Japan. This work was supported in part by the US National Science Foundation under award PHY-1316617.

\section{A Conventions, definitions, and identities}

In this work we denote $10 \mathrm{~d}$ spacetime indices by capital Latin letters $M, N=0, \ldots, 9,4 \mathrm{~d}$ spacetime indices by $\mu, \nu=0,1,2,3$, and the internal complex indices by $m, n, p=1, \ldots, 3$ and $\bar{m}, \bar{n}, \bar{p}=1, \ldots, 3$. We occasionally also make use of real internal indices $a, b=1, \ldots, 6$. The metric signature of the ten-dimensional space is $(-,+, \ldots,+)$. Our conventions for the totally anti-symmetric tensor in Lorentzian signature in an orthonormal frame are $\epsilon_{012 \ldots 9}=\epsilon_{0123}=+1$. The epsilon tensor in $d$ dimensions then satisfies

$$
\left.\epsilon^{R_{1} \cdots R_{p} N_{1} \ldots N_{d-p}} \epsilon_{R_{1} \ldots R_{p} M_{1} \ldots M_{d-p}}=(-1)^{s}(d-p) ! p ! \delta^{N_{1}}{ }_{\left[M_{1}\right.} \ldots \delta^{N_{d-p}} M_{d-p}\right],
$$

where $s=0$ if the metric has Euclidean signature and $s=1$ for a Lorentzian metric.

We adopt the following conventions for the Christoffel symbols and Riemann tensor

$$
\begin{aligned}
\Gamma^{R}{ }_{M N} & =\frac{1}{2} g^{R S}\left(\partial_{M} g_{N S}+\partial_{N} g_{M S}-\partial_{S} g_{M N}\right), & R_{M N} & =R_{M R N}^{R}, \\
R_{N R S}^{M} & =\partial_{R} \Gamma_{S N}^{M}-\partial_{S} \Gamma_{R N}^{M}+\Gamma_{R T}^{M} \Gamma_{S N}^{T}-\Gamma_{S T}^{M} \Gamma_{R N}^{T}, & R & =R_{M N} g^{M N}
\end{aligned}
$$


with equivalent definitions on the internal and external spaces. Differential $p$-forms are expanded in a basis of differential one-forms as

$$
\Lambda=\frac{1}{p !} \Lambda_{M_{1} \ldots M_{p}} d x^{M_{1}} \wedge \ldots \wedge d x^{M_{p}} .
$$

The wedge product between a $p$-form $\Lambda^{(p)}$ and a $q$-form $\Lambda^{(q)}$ is given by

$$
\left(\Lambda^{(p)} \wedge \Lambda^{(q)}\right)_{M_{1} \ldots M_{p+q}}=\frac{(p+q) !}{p ! q !} \Lambda_{\left[M_{1} \ldots M_{p}\right.}^{(p)} \Lambda_{\left.M_{1} \ldots M_{q}\right]}^{(q)} .
$$

Furthermore, the exterior derivative on a $p$-form $\Lambda$ reads

$$
(d \Lambda)_{N M_{1} \ldots M_{p}}=(p+1) \partial_{[N} \Lambda_{\left.M_{1} \ldots M_{p}\right]},
$$

while the Hodge star of $p$-form $\Lambda$ in $d$ real coordinates is given by

$$
\left(*_{d} \Lambda\right)_{N_{1} \ldots N_{d-p}}=\frac{1}{p !} \Lambda^{M_{1} \ldots M_{p}} \epsilon_{M_{1} \ldots M_{p} N_{1} \ldots N_{d-p}} .
$$

Moreover, the identity

$$
\Lambda^{(1)} \wedge * \Lambda^{(2)}=\frac{1}{p !} \Lambda_{M_{1} \ldots M_{p}}^{(1)} \Lambda^{(2) M_{1} \ldots M_{p}} * 1
$$

holds for two arbitrary $p$-forms $\Lambda^{(1)}$ and $\Lambda^{(2)}$.

Let us specify in more detail our conventions regarding complex coordinates in the internal space. For a complex manifold $M$ with complex dimension $n$ the complex coordinates $z^{1}, \ldots, z^{n}$ and the underlying real coordinates $y^{1}, \ldots, y^{2 n}$ are related by

$$
\left(z^{1}, \ldots, z^{n}\right)=\left(\frac{1}{\sqrt{2}}\left(y^{1}+i y^{2}\right), \ldots, \frac{1}{\sqrt{2}}\left(y^{2 n-1}+i y^{2 n}\right)\right) .
$$

Using these conventions one finds

$$
\sqrt{g} d y^{1} \wedge \ldots \wedge d y^{2 n}=\sqrt{g}(-1)^{\frac{(n-1) n}{2}} i^{n} d z^{1} \wedge \ldots \wedge d z^{n} \wedge d \bar{z}^{1} \wedge \ldots \wedge d \bar{z}^{n}=\frac{1}{n !} J^{n},
$$

with $g$ the determinant of the metric $g_{a b}$ in real coordinates and $\sqrt{\operatorname{det} g_{a b}}=\operatorname{det} g_{m \bar{n}}$. The Kähler form is given by

$$
J=i g_{m \bar{n}} d z^{m} \wedge d \bar{z}^{\bar{n}} .
$$

Let $\omega_{p, q}$ be a $(p, q)$-form, then its Hodge dual is the $(n-q, n-p)$ form

$$
\begin{aligned}
* \omega_{p, q}= & \frac{(-1)^{\frac{n(n-1)}{2}} i^{n}(-1)^{p n}}{p ! q !(n-p) !(n-q) !} \omega_{m_{1} \ldots m_{p} \bar{n}_{1} \ldots \bar{n}_{q}} \epsilon^{m_{1} \ldots m_{p}} \bar{r}_{1} \ldots \bar{r}_{n-p} \epsilon_{s_{1} \ldots s_{n-q}}^{\bar{n}_{1} \ldots \bar{n}_{q}} \times \\
& \times d z^{s_{1}} \wedge \cdots \wedge d z^{s_{n-q}} \wedge d \bar{z}^{\bar{r}_{1}} \wedge \cdots \wedge d \bar{z}^{n-p} .
\end{aligned}
$$

Finally, let us record our conventions regarding Chern forms. To begin with, we define the curvature two-form for Hermitian manifolds to be

$$
\mathcal{R}^{m}{ }_{n}=R^{m}{ }_{n r \bar{s}} d z^{r} \wedge d \bar{z}^{\bar{s}}
$$


and we set

$$
\begin{aligned}
& \operatorname{Tr} \mathcal{R}=R^{m}{ }_{m r_{1} \bar{s}_{2}} d z^{r_{1}} \wedge d \bar{z}^{\bar{s}_{1}} \\
& \operatorname{Tr} \mathcal{R}^{2}=R^{m}{ }_{n r_{1} \bar{s}_{1}} R^{n}{ }_{m r_{2} \bar{s}_{2}} d z^{r_{1}} \wedge d \bar{z}^{\bar{s}_{1}} \wedge d z^{r_{2}} \wedge d \bar{z}^{\bar{s}_{2}}, \\
& \operatorname{Tr} \mathcal{R}^{3}=R^{m}{ }_{n r_{1} \bar{s}_{1}} R^{n}{ }_{p r_{2} \bar{s}_{2}} R^{p}{ }_{m r_{3} \bar{s}_{3}} \wedge d z^{r_{1}} \wedge d \bar{z}^{\bar{s}_{1}} \wedge d z^{r_{2}} \wedge d \bar{z}^{\bar{s}_{2}} \wedge d z^{r_{3}} \wedge d \bar{z}^{\bar{s}_{3}} .
\end{aligned}
$$

The Chern forms can then be expressed in terms of the curvature two-form as

$$
\begin{aligned}
& c_{0}=1 \\
& c_{1}=\frac{1}{2 \pi} i \operatorname{Tr} \mathcal{R}, \\
& c_{2}=\frac{1}{(2 \pi)^{2}} \frac{1}{2}\left(\operatorname{Tr} \mathcal{R}^{2}-(\operatorname{Tr} \mathcal{R})^{2}\right), \\
& c_{3}=\frac{1}{3} c_{1} c_{2}+\frac{1}{(2 \pi)^{2}} \frac{1}{3} c_{1} \wedge \operatorname{Tr} \mathcal{R}^{2}-\frac{1}{(2 \pi)^{3}} \frac{i}{3} \operatorname{Tr} \mathcal{R}^{3}, \\
& c_{4}=\frac{1}{24}\left(c_{1}^{4}-\frac{1}{(2 \pi)^{2}} 6 c_{1}^{2} \operatorname{Tr} \mathcal{R}^{2}-\frac{1}{(2 \pi)^{3}} 8 i c_{1} \operatorname{Tr} \mathcal{R}^{3}\right)+\frac{1}{(2 \pi)^{4}} \frac{1}{8}\left(\left(\operatorname{Tr} \mathcal{R}^{2}\right)^{2}-2 \operatorname{Tr} \mathcal{R}^{4}\right) .
\end{aligned}
$$

The Chern forms of an $n$-dimensional Calabi-Yau manifold $Y_{n}$ reduce to

$$
c_{3}\left(Y_{n \geq 3}\right)=-\frac{1}{(2 \pi)^{3}} \frac{i}{3} \operatorname{Tr} \mathcal{R}^{3} \text { and } c_{4}\left(Y_{n \geq 4}\right)=\frac{1}{(2 \pi)^{4}} \frac{1}{8}\left(\left(\operatorname{Tr} \mathcal{R}^{2}\right)^{2}-2 \operatorname{Tr} \mathcal{R}^{4}\right) .
$$

\section{B Higher-derivative dilaton terms}

In this appendix we record the expression of the quantities $f_{1}, f_{2}^{M N}, f_{3}$ introduced in (2.9). We have

$$
\begin{aligned}
& f_{1}=192 R_{M}{ }^{R} O^{S} R^{M N O P} R_{N S P R}-48 R_{M N}{ }^{R S} R^{M N O P} R_{O P R S} \\
& +576 R^{M N}{ }_{M}^{O} R_{N}{ }^{P R S} R_{O P R S}+384 R^{M N}{ }_{M}{ }^{O} R_{N}{ }^{P}{ }_{P}{ }^{R} R_{O}{ }_{R S} \\
& -72 R^{M N}{ }_{M N} R_{O P R S} R^{O P R S}-576 R^{M N}{ }_{M}{ }^{O} R_{N}{ }^{P}{ }_{O}{ }^{R} R_{P}{ }^{S} R S \\
& +288 R^{M N}{ }_{M N} R^{O P}{ }_{O}{ }^{R} R_{P}{ }_{R S}-24 R^{M N}{ }_{M N} R^{O P}{ }_{O P} R^{R S}{ }_{R S} \text {, } \\
& f_{2}^{M N}=g^{M N}\left(192 R_{R}{ }^{Q}{ }_{O}^{V} R^{R S O P} R_{S V P Q}-48 R_{R S}{ }^{Q V} R^{R S O P} R_{O P Q V}\right. \\
& +576 R^{R S}{ }_{R}^{O} R_{S}{ }^{P Q V} R_{O P Q V}+384 R^{R S}{ }_{R}{ }^{O} R_{S}{ }^{P}{ }_{P}{ }^{Q} R_{O}{ }^{V} Q V \\
& -72 R^{R S}{ }_{R S} R_{O P Q V} R^{O P Q V}-576 R^{R S}{ }_{R}{ }^{O} R_{S}{ }^{P}{ }_{O}{ }^{Q} R_{P}{ }^{V} Q V \\
& \left.+288 R^{R S}{ }_{R S} R^{O P}{ }_{O}^{Q} R_{P}{ }_{Q V}-24 R^{R S}{ }_{R S} R^{O P}{ }_{O P} R^{Q V}{ }_{Q V}\right)+\cdots=g^{M N} f_{1}+\ldots
\end{aligned}
$$

where the ellipsis denote terms where the free indices $M, N$ are on the Riemann tensors and thus will not contribute to our discussion. Furthermore,

$$
\begin{aligned}
f_{3}= & 768 R_{M O}{ }^{R S} R^{M N O P} R_{N R P S}+384 R_{M}{ }^{R}{ }^{S} R^{M N O P} R_{N S P R} \\
& -96 R_{M N}{ }^{R S} R^{M N O P} R_{O P R S}+1536 R^{M N}{ }_{M}{ }^{O} R_{N}{ }^{P R S} R_{O P R S} \\
& -384 R_{M N}{ }^{R S} R^{M N O P} R_{O R P S}+768 R^{M N}{ }_{M}{ }^{O} R_{N}{ }^{P R S} R_{O R P S} \\
& +1536 R^{M N}{ }_{M}{ }^{O} R_{N}{ }^{P}{ }_{P}{ }^{R} R_{O}{ }^{S}{ }_{R S}-240 R^{M N}{ }_{M N} R_{O P R S} R^{O P R S} \\
& -1920 R^{M N}{ }_{M}{ }^{O} R_{N}{ }^{P}{ }_{O}{ }^{R} R_{P}{ }^{S}{ }_{R S}+1152 R^{M N}{ }_{M N} R^{O P}{ }_{O}{ }^{R} R_{P}{ }_{R S} \\
& -96 R^{M N}{ }_{M N} R^{O P}{ }_{O P} R^{R S}{ }_{R S} .
\end{aligned}
$$


Open Access. This article is distributed under the terms of the Creative Commons Attribution License (CC-BY 4.0), which permits any use, distribution and reproduction in any medium, provided the original author(s) and source are credited.

\section{References}

[1] M. Graña, Flux compactifications in string theory: A Comprehensive review, Phys. Rept. 423 (2006) 91 [hep-th/0509003] [INSPIRE].

[2] M.R. Douglas and S. Kachru, Flux compactification, Rev. Mod. Phys. 79 (2007) 733 [hep-th/0610102] [InSPIRE].

[3] R. Blumenhagen, B. Körs, D. Lüst and S. Stieberger, Four-dimensional String Compactifications with D-branes, Orientifolds and Fluxes, Phys. Rept. 445 (2007) 1 [hep-th/0610327] [INSPIRE].

[4] F. Denef, M.R. Douglas and S. Kachru, Physics of String Flux Compactifications, Ann. Rev. Nucl. Part. Sci. 57 (2007) 119 [hep-th/0701050] [InSPIRE].

[5] H. Samtleben, Lectures on Gauged Supergravity and Flux Compactifications, Class. Quant. Grav. 25 (2008) 214002 [arXiv:0808.4076] [INSPIRE].

[6] K. Becker, M. Becker, M. Haack and J. Louis, Supersymmetry breaking and alpha-prime corrections to flux induced potentials, JHEP 06 (2002) 060 [hep-th/0204254] [INSPIRE].

[7] V. Balasubramanian, P. Berglund, J.P. Conlon and F. Quevedo, Systematics of moduli stabilisation in Calabi-Yau flux compactifications, JHEP 03 (2005) 007 [hep-th/0502058] [INSPIRE].

[8] J.P. Conlon, F. Quevedo and K. Suruliz, Large-volume flux compactifications: Moduli spectrum and D3/D7 soft supersymmetry breaking, JHEP 08 (2005) 007 [hep-th/0505076] [INSPIRE].

[9] M. Cicoli, J.P. Conlon and F. Quevedo, General Analysis of LARGE Volume Scenarios with String Loop Moduli Stabilisation, JHEP 10 (2008) 105 [arXiv:0805.1029] [INSPIRE].

[10] M.T. Grisaru, A.E.M. van de Ven and D. Zanon, Two-Dimensional Supersymmetric $\sigma$-models on Ricci Flat Kähler Manifolds Are Not Finite, Nucl. Phys. B 277 (1986) 388 [InSPIRE].

[11] M.T. Grisaru, A.E.M. van de Ven and D. Zanon, Four Loop Divergences for the $N=1$ Supersymmetric Nonlinear $\sigma$-model in Two-Dimensions, Nucl. Phys. B 277 (1986) 409 [INSPIRE].

[12] J.H. Schwarz, Superstring Theory, Phys. Rept. 89 (1982) 223 [InSPIRE].

[13] D.J. Gross and E. Witten, Superstring Modifications of Einstein's Equations, Nucl. Phys. B 277 (1986) 1 [INSPIRE].

[14] D.J. Gross and J.H. Sloan, The Quartic Effective Action for the Heterotic String, Nucl. Phys. B 291 (1987) 41 [INSPIRE].

[15] N. Sakai and Y. Tanii, One Loop Amplitudes and Effective Action in Superstring Theories, Nucl. Phys. B 287 (1987) 457 [InSPIRE].

[16] M. Abe, H. Kubota and N. Sakai, Loop Corrections to the Heterotic String Effective Lagrangian, Phys. Lett. B 200 (1988) 461 [INSPIRE]. 
[17] A. Kehagias and H. Partouche, On the exact quartic effective action for the type IIB superstring, Phys. Lett. B 422 (1998) 109 [hep-th/9710023] [INSPIRE].

[18] A. Kehagias and H. Partouche, D instanton corrections as $(p, q)$ string effects and nonrenormalization theorems, Int. J. Mod. Phys. A 13 (1998) 5075 [hep-th/9712164] [INSPIRE].

[19] R. Minasian, T.G. Pugh and R. Savelli, F-theory at order $\alpha^{\prime 3}$, JHEP 10 (2015) 050 [arXiv: 1506.06756] [INSPIRE].

[20] S. Ferrara and S. Sabharwal, Quaternionic Manifolds for Type II Superstring Vacua of Calabi-Yau Spaces, Nucl. Phys. B 332 (1990) 317 [inSPIRE].

[21] P. Candelas, X.C. De La Ossa, P.S. Green and L. Parkes, A Pair of Calabi-Yau manifolds as an exactly soluble superconformal theory, Nucl. Phys. B 359 (1991) 21 [INSPIRE].

[22] R. Bohm, H. Gunther, C. Herrmann and J. Louis, Compactification of type IIB string theory on Calabi-Yau threefolds, Nucl. Phys. B 569 (2000) 229 [hep-th/9908007] [INSPIRE].

[23] D. Joyce, Lectures on Calabi-Yau and special Lagrangian geometry, math/0108088 [INSPIRE].

[24] T.W. Grimm, T.G. Pugh and M. Weissenbacher, On M-theory fourfold vacua with higher curvature terms, Phys. Lett. B 743 (2015) 284 [arXiv: 1408.5136] [INSPIRE].

[25] T.W. Grimm, T.G. Pugh and M. Weissenbacher, The effective action of warped M-theory reductions with higher derivative terms — part I, JHEP 01 (2016) 142 [arXiv:1412.5073] [INSPIRE].

[26] T.W. Grimm, T.G. Pugh and M. Weissenbacher, The effective action of warped M-theory reductions with higher-derivative terms - Part II, JHEP 12 (2015) 117 [arXiv:1507.00343] [INSPIRE].

[27] T.W. Grimm, R. Savelli and M. Weissenbacher, On $\alpha^{\prime}$ corrections in $N=1$ F-theory compactifications, Phys. Lett. B $\mathbf{7 2 5}$ (2013) 431 [arXiv:1303.3317] [INSPIRE].

[28] T.W. Grimm, J. Keitel, R. Savelli and M. Weissenbacher, From M-theory higher curvature terms to $\alpha^{\prime}$ corrections in F-theory, Nucl. Phys. B 903 (2016) 325 [arXiv:1312.1376] [INSPIRE].

[29] M. Weissenbacher, On four-derivative terms in IIB Calabi-Yau orientifold reductions, arXiv: 1607.03913 [INSPIRE].

[30] T.W. Grimm and J. Louis, The Effective action of $N=1$ Calabi-Yau orientifolds, Nucl. Phys. B 699 (2004) 387 [hep-th/0403067] [INSPIRE].

[31] T.W. Grimm and J. Louis, The Effective action of type IIA Calabi-Yau orientifolds, Nucl. Phys. B 718 (2005) 153 [hep-th/0412277] [INSPIRE].

[32] T.W. Grimm, The Effective action of type-II Calabi-Yau orientifolds, Fortsch. Phys. 53 (2005) 1179 [hep-th/0507153] [INSPIRE].

[33] G. Policastro and D. Tsimpis, $R^{4}$, purified, Class. Quant. Grav. 23 (2006) 4753 [hep-th/0603165] [INSPIRE].

[34] G. Policastro and D. Tsimpis, A Note on the quartic effective action of type IIB superstring, Class. Quant. Grav. 26 (2009) 125001 [arXiv:0812.3138] [INSPIRE].

[35] S.B. Giddings, S. Kachru and J. Polchinski, Hierarchies from fluxes in string compactifications, Phys. Rev. D 66 (2002) 106006 [hep-th/0105097] [INSPIRE]. 
[36] D. Nemeschansky and A. Sen, Conformal Invariance of Supersymmetric $\sigma$ Models on Calabi-Yau Manifolds, Phys. Lett. B 178 (1986) 365 [InSPIRE].

[37] M.D. Freeman and C.N. Pope, $\beta$-functions and Superstring Compactifications, Phys. Lett. B 174 (1986) 48 [INSPIRE].

[38] M.D. Freeman, C.N. Pope, M.F. Sohnius and K.S. Stelle, Higher Order $\sigma$ Model Counterterms and the Effective Action for Superstrings, Phys. Lett. B 178 (1986) 199 [INSPIRE].

[39] P. Candelas, M.D. Freeman, C.N. Pope, M.F. Sohnius and K.S. Stelle, Higher Order Corrections to Supersymmetry and Compactifications of the Heterotic String, Phys. Lett. B 177 (1986) 341 [INSPIRE].

[40] I. Antoniadis, S. Ferrara, R. Minasian and K.S. Narain, $R^{4}$ couplings in $M$ and type-II theories on Calabi-Yau spaces, Nucl. Phys. B 507 (1997) 571 [hep-th/9707013] [INSPIRE].

[41] T. House and E. Palti, Effective action of (massive) IIA on manifolds with SU(3) structure, Phys. Rev. D 72 (2005) 026004 [hep-th/0505177] [InSPIRE].

[42] D. Andriot, New supersymmetric vacua on solvmanifolds, JHEP 02 (2016) 112 [arXiv: 1507.00014] [INSPIRE].

[43] P. Candelas, X.C. De la Ossa, P.S. Green and L. Parkes, An Exactly soluble superconformal theory from a mirror pair of Calabi-Yau manifolds, Phys. Lett. B 258 (1991) 118 [INSPIRE].

[44] S. Ferrara and S. Sabharwal, Dimensional Reduction of Type II Superstrings, Class. Quant. Grav. 6 (1989) L77 [InSPIRE].

[45] M. Bodner and A.C. Cadavid, Dimensional Reduction of Type IIB Supergravity and Exceptional Quaternionic Manifolds, Class. Quant. Grav. 7 (1990) 829 [INSPIRE].

[46] S. Hosono, A. Klemm and S. Theisen, Lectures on mirror symmetry, Lect. Notes Phys. 436 (1994) 235 [hep-th/9403096] [INSPIRE].

[47] K. Hori et al., Clay mathematics monographs. Vol. 1: Mirror symmetry, AMS, Providence, U.S.A. (2003).

[48] D. Ciupke and L. Zarate, Classification of Shift-Symmetric No-Scale Supergravities, JHEP 11 (2015) 179 [arXiv:1509.00855] [INSPIRE].

[49] S. Kachru, R. Kallosh, A.D. Linde and S.P. Trivedi, de Sitter vacua in string theory, Phys. Rev. D 68 (2003) 046005 [hep-th/0301240] [InSPIRE]. 